\title{
Measurement, Information Channels, and Discretization: Exploring the Links
}

\author{
(Invited Article)
}

\author{
Zoltan Domotor $^{1 *}$ and Vadim Batitsky ${ }^{2}$ \\ ${ }^{1}$ Departments of Philosophy, Biochemistry and Biophysics, University of Pennsylvania, Philadelphia, PA 19104, USA \\ ${ }^{2}$ Department of Philosophy, St. John's University, Queens, NY 11439, USA
}

The goal of this paper is to present a unified algebraic-analytic framework for (static and dynamic) deterministic measurement theory, which we find to be fully adequate in engineering and natural science applications. The starting point of this paradigm is the notion of a quantity algebra of a measured system and that of a measuring instrument, underlying the causal linkages in classical 'system + instrument' interactions. This approach is then further enriched by providing a superimposed data lattice of measurement outcomes, intended to handle the information flow from the measured system to its measurand's designated instrument.

We argue that the language of Banach and von Neumann algebras is ideally suited for the treatment of quantities, encountered in theoretical and experimental science. These algebras and convex spaces of expectation functionals thereon together with information (co)channels between them provide a comprehensive information-theoretic framework for measurement theory. Concrete examples and applications to length and position measurements are also discussed and rigorously framed within the proposed quantity algebra and associated information channel paradigms.

In modeling physical systems, investigators routinely rely on the assumption that state spaces and time domains form a continuum (locally homeomorphic to the real line or its Cartesian powers). But in sharp contrast, measurement and prediction outcomes pertaining to physical systems under consideration tend to be presented in terms of small discrete sets of rational numbers. We investigate this conceptual gap between theoretical and finitary data models from the perspectives of temporal, spatial and algebraic discretization schemes.

The principal innovation in our approach to classical measurement theory is the representation of interactive instrument-based measurement processes in terms of channel-cochannel pairs constructed between dynamical quantity algebras of a target system and its measurand's measuring instrument. tion

Keywords: quantity algebra, measurement theory, instrument model, information channel, cochannel, discretiza-

\section{INTRODUCTION AND BACKGROUND}

$I^{N}$ THE NATURAL SCIENCES, engineering, and technological applications, the aim of a measurement process is to obtain verifiable numerical information about the target system's extant state. Partial information about the system's state at a given site and moment of time is made available by measuring the system's designated quantity (i.e., a pre-existing quantitative attribute to be measured) - called a measurandat that site and time. The measurement process involves the determination of the value of a suitable pointer quantity, which has become maximally correlated to the measurand's actual value, thanks to a direct physical interaction between the target system and the measurand's measuring instrument - designed for reading the values of its pointer quantity. Information about the system's state together with the system's validated theoretical model can be used in predicting some of the results of future measurements.

The algebraic-analytic framework for measurement theory

*Corresponding author: zdomotor@sas.upenn.edu presented in [4] approached measurement as an interaction between a target (measured) system and a measuring instrument, designed and calibrated to measure some specific quantity instantiated by the target system. With the target system and the measuring instrument modeled by their respective quantity algebras (formally Banach algebras), the main aspects of deterministic measurement were modeled by natural algebraic constructions, such as tensor products of quantity algebras (representing the compound 'target+ measuring' system), temporal dynamics on tensor products of quantity algebras (representing the dynamical measurement coupling of the target system and the measuring instrument), and more. It was also shown how a fundamental theorem in analysis, known as Gelfand representation, guarantees that the syntactic framework of autonomously specified quantity algebras (and various constructions effected from them) comes with a realist semantics (interpretation) on which autonomously specified quantity algebras can be identyfied with concrete algebras of observables on uniquely determined topological spaces of the kind commonly used by scientists as state 
spaces of natural systems in the world.

Because the initial presentation of this algebraic-analytic framework was only brought up as a viable alternative to the representational theory of measurement, several epistemologically and methodologically important issues pertaining to real world measurements remained unexplored. These issues will be addressed in the present paper, which enriches and refines the framework of Banach algebras, so as to obtain conceptually and formally adequate models of the following aspects of measurement:

(i) Error and uncertainty in measurement: Because real world measuring instruments do not possess unlimited degrees of accuracy, all scientific measurements are subject to uncertainty in general, and deterministic and random errors in particular. The inherent element of randomness and uncertainty, however, is not reflected in the framework of Banach algebras, where quantities are presumed to be smooth or continuous, and where measurements are treated as ideally perfect determinations of such quantities' values. To make our algebraic models of measurement epistemologically and methodologically more realistic, we will introduce a mathematically broader notion of measurable (random) quantities, and will use von Neumann algebras as our quantity algebras for modeling measurement interactions whose results are known only to the extent that they belong to certain measurable sets of reals with such-and-such probability. Analogously to the earlier mentioned Gelfand representation for Banach algebras, we will show that another theorem in analysis, known as Riesz representation, provides us with a semantic interpretation of autonomously specified von Neumann algebras in terms of concrete von Neumann algebras of random variables on suitable measure spaces.

(ii) Relations between infinite/continuum theoretical models and finite/discrete measurement data: Theoretical science relies extensively on modeling natural phenomena using families of differential equations over realor complex-valued functions (based on the ontological assumption that the underlying domains of time, space and system states are furnished with the structure of a topological continuum). On the other hand, real-world measurements can yield only finite amounts of information about a target natural system. As a result, quantities in theoretical models may take on numerical values which cannot be experimentally determined with any fixed finite amount of measurement and computational resources (e.g., in the case of transcendental reals), or even in the limit of infinite precision allowed by highly idealized measuring instruments (if the measurand's values are non-computable or random reals). A theory of measurement, we believe, should explore this gap between the ontologically motivated continuum-based theoretical models and our resource-bounded epistemic access to the modeled natural systems through finite and discrete measurement data. To this end, the present paper will follow [2] by introducing the notion of (spatial and temporal) discretization of continuum dynamical models, and investigate the formal relationships between continuum theoretical models and their discretized versions in the context of measurement interactions. Along the way, we also exploit the fact that Gelfand and Riesz representations - which relate dynamical algebras and topological dynamical models - also holds for discretized versions of these algebraic and topological dynamical models.

(iii) Information channel perspective on measurement: As a matter of scientific practice, a given quantity instantiated by a target natural system can be measured in different ways, with varying degrees of accuracy, using different measuring instruments, different background laws involved in the design and calibration of measuring instruments, and so on. Yet, despite being quite different qua physical interactions, such measurements have something in common which makes them all measurements of one and the same quantity. Clearly, this common property shared by physically distinct methods of measuring a given quantity cannot be captured at the physical level of description, except by trivial disjunctive descriptions of all the different physical processes underlying such measurements. On the other hand, this common property can be handled at a 'higher' (conceptually more general) level, called a functionalist perspective on measurement - the perspective which abstracts measurement away from its specific underlying physical processes and allows us to view it as a transfer of information about the measurand from its instantiating target system to the instrument's pointer module. The motivation for this functionalist perspective on measurement is quite analogous to that behind the machine-independent perspective on computability, which abstracts away from specific equivalent models of computation (e.g., a Turing machine, Post production system, Markov algorithm, etc.) and focuses on the general properties of computable functions. In this paper we will explore several formal and conceptual advantages of this functionalist information-theoretic perspective on measurement by introducing and studying the notion of a channel as a formal model of the causally directed transfer of information about the measurand to the measuring instrument. Of special importance to this investigation will be a theorem known as Umegaki representation, which guarantees that every information channel target system $\longrightarrow$ measuring instrument comes with its dual conversely directed cochannel: measuring instrument $\longrightarrow$ target system. The latter will be used in modeling the estimation (reconstruction) of the measurand's objective value from the measuring instrument's output.

By way of concluding this introduction, we would like to emphasize that the rather technical algebraic-analytic ap- 
proach to measurement presented in this paper is motivated by (and ultimately gives support to) our philosophical commitment to the realist conception of quantities - the conception which opposes the instrumentalist view of quantities underlying the well-known representational theory of measurement, and on which (i) quantities are theoretical entities of science interpreted as objective characteristics of natural systems in the world, and (ii) measurement is a physical process of (approximate) determination or estimation of the value posed by a quantity under consideration, as instantiated by the target natural system.

\section{AlgEBRAIC-ANALYTIC FRAMEWORK FOR CLASSICAL MEASUREMENT THEORY}

In this section we recall the general mathematical structure of classical physical measurement we shall need. Instrumentbased measurement of a quantity of interest (e.g., electric current, volume, temperature, mechanical stress, X-ray energy, etc.), commonly called a measurand, is a physical process involving a target system that (i) instantiates the measurand in a particular amount or degree, (ii) is dynamically coupled to the measurand's designated measuring instrument during the act of measurement, and (iii) contingently upon its state, the system sends information about the measurand's extant value via the composite 'system + instrument' bipartite system to the instrument's pointer module, ready for readout.

Although the formal structure of much of classical physics and systems science is nothing more than a suitable dynamics (traditionally described by differential or difference equations) and a statistics of quantities, the mathematical framework for physical measurement processes ordinarily involves the following three major algebraic-analytic and probabilistic ingredients: (1) quantity algebras together with their (deterministic and probabilistic) state spaces, independently representing the target system and the measurand's measuring instrument, (2) joint physical dynamics of the 'system + instrument' compound system, and (3) a supervening information channel from the system's state space to that of the instrument's pointer module. To see the details, we need to recall some basic concepts pertaining to mathematical measurement theory. We start with the characterization of quantity algebras and accompanying state spaces.

\subsection{Quantity algebras of measured and measuring systems}

Here we follow the algebraic-analytic framework for measurement theory, outlined in [4] and further developed in [5]. It is impracticable to study classical physical measurement without an explicit commitment to appropriate (continuous, measurable, smooth, discretized, etc.) quantities, such as energy, force, electric charge, pressure, and so forth, and lawlike relations between them. Accordingly, the starting point of measurement modeling in engineering and the natural sciences is the specification of a physically relevant algebra $\mathfrak{A}_{\mathrm{S}}$ of quantities that is believed to correctly describe the quan- titative attributes and behavior of the natural system $S$ under consideration. For reasons of mathematical tractability, the quantity algebra can not be exhaustively detailed. Many aspects of the natural system of interest are deliberately omitted, suppressed or enhanced. The system's quantity algebra is not intended to be an exact mathematical copy of reality. Instead, it is constructed with special regards to formulating scientific concepts and ideas that facilitate the model builder's understanding of the system's structure and function. In practice, the algebra is generated by a remarkably short list of quantities (e.g., positions and momenta of point particles or pressure, volume and thermodynamic temperature of gases in vessels, or currents and potential differences in electric circuits) that meets the aims of quantitative modeling of measurement at a given level of idealization and simplification. The discrepancy between the degrees of freedom present in the target natural system $S$ and those captured by the representing quantity algebra $\mathfrak{A}_{\mathrm{S}}$ inevitably leads to various modeling errors. This is not a serious problem as long as the selected algebra is sufficiently close to the actual system in the sense that the differences between measurement results and model-generated predictions (and explanations) that could tell them apart are well within the bounds of antecedently specified admissible errors. Overidealization or overabstraction embodied in the system's quantity algebra $\mathfrak{A}_{\mathrm{S}}$ can lead not only to information reduction in the sense that not all quantities or behaviors of interest are faithfully encoded in its structure, but also to information distortion, meaning that the algebra allows behaviors which are not present in the natural system under consideration. This is the price a modeler has to pay for incorrectly handling system complexity and for failing to delineate the model's domain of applicability.

In practice, all measurands which are believed to be decisive in the description of the target system's structure and behavior of interest are automatically included in its associated quantity algebra $\mathfrak{A}$. To uphold the standard laws of addition of quantities of the same physical dimension and those of distributive multiplication relied upon in secondary quantities (such as density, pressure, stress, and so on), the algebra $\mathfrak{A}$ is presumed to have the structure of a unital commutative ring. In particular, for any pair of quantities $f, g$ in $\mathfrak{A}$ we have their associative and reversible addition $f+g$, and a bilinear, associative and commutative multiplication $f \bullet g$ with a unique (constant) unit quantity 1 satisfying $l \bullet f=f \bullet l=f$, all in $\mathfrak{A}$. Rational scale changes of quantities prompt to include the operation of scalar multiplication, which assigns to each quantity $f$ in $\mathfrak{A}$ and a scalar $c$ in the field of reals $\mathbb{R}$ the unique scalar product quantity, denoted $c \cdot f$, again in $\mathfrak{A}$. In this way, the quantity algebra is automatically also a linear space over $\mathbb{R}$. In view of topological and measure-theoretic properties of many quantitative attributes arising in physics and engineering, quantity algebras tend to come with a norm, i.e., a non-negative real-valued function $\|\cdot\|: \mathfrak{A} \longrightarrow \mathbb{R}$ satisfying the usual norm axioms and the inequality $\|f \bullet g\| \leq\|f\| \cdot\|g\|$ for all $f, g \in \mathfrak{A}$. A topologically complete, normed, real, unital, commutative algebra described above is important in its 
own right and therefore has a special name - it is called a real (unital, commutative) Banach algebra. It is good to bear in mind that there is no proof that the algebraic language of Banach algebras is fully universal in the sense that within it all mainline physical measurements of quantities can be framed. What we can claim, however, is that all known empirically adequate theories of classical measurement processes involving continuous quantities admit a Banach algebra formulation. Of special appeal is the fact that noncommutative Banach algebras over the immutable complex field provide an established framework for reasoning about quantum measurement and, moreover, certain assignments of appropriate Banach algebras to space-time regions are well suited to the study of relativistic mechanical and electromagnetic field measurements. Banach algebras are furnished with as much algebraic and geometric structure as a measurement theorist could ever wish for in her theory. We now extend the foregoing primer on Banach algebras with a few facts on probabilistic states thereon and channels between them.

\subsubsection{Banach algebra approach to physical measure- ment}

We devote this part of Subsection 2.1 to setting down, in precise terms, the definitions of states, and information channels between the state spaces of measured and measuring systems. In the second part we shall confine our attention to the properties of von Neumann algebras of measurable quantities and their Riesz representation.

In support of dimensional analysis, some quantities $f$ (e.g., volume and area, needed for defining density and pressure, respectively) are presumed to come with a unique inverse quantity $f^{-1}$, satisfying the equalities $f \bullet f^{-1}=f^{-1} \bullet f=1$ and $(f \bullet g)^{-1}=f^{-1} \bullet g^{-1}$, if both $f$ and $g$ are invertible. It is easy to check that the set of invertible quantities (i.e., the ones that possess an inverse) in a Banach algebra $\mathfrak{A}$ is a group with respect to the multiplication operation $\bullet$, conveniently denoted by $\mathrm{Gr} \mathfrak{A}$. Importantly, each quantity $f$ in $\mathfrak{A}$ has a unique value (spectral) space

$$
\operatorname{Val}(f)={ }_{d f}\{c \in \mathbb{R} \mid c \cdot 1-f \notin \mathrm{Gr} \mathfrak{A}\}
$$

that abstractly (i.e., without any evaluation device) specifies the quantity's set of possible numerical values. The value space captures the standard lore about quantities as attributes possessing values that match their concrete instantiations in degrees or amounts. Specifically, if experimenters were to measure measurand $f$, ideally they would obtain exactly one of the values in $\operatorname{Val}(f)$ as the measurement outcome. However, as everybody in the trade knows, the measurement outcome in a normal real-world measurement process is at best only the value of the measuring instrument's pointer quantity $\oslash f$ (chosen for measuring $f$ ) that is presumed to correctly approximate the actual value of $f$.

A good mathematical source of examples of Banach algebras are the rings of continuous real-valued functions on a topological space. Concretely, if $\mathscr{X}$ is a compact Hausdorff space, then the space $\mathbf{C}(\mathscr{X})$ of all continuous real-valued functions of the form $f: \mathscr{X} \longrightarrow \mathbb{R}$ is a real (unital, commutative) Banach algebra under pointwise-defined algebraic operations (inherited from $\mathbb{R}$ ) and the supremum norm. In this case it is elementary to verify that the value space $\operatorname{Val}(f)$ of $f$ equals to its numerical range $\{f(x) \mid x \in \mathscr{X}\} \subseteq \mathbb{R}$. Quantities of a more complex type (e.g., vector and tensor quantities) come with values belonging to $\mathbb{R}^{n}$ (for some natural number $n$ ) or other more involved sets constructed from the host ground field $\mathbb{R}$ of reals.

We mentioned that most quantity algebras arising in applications tend to be finitely generated. Recall that a finite subset of linearly independent quantities, say, $\{f, g\}$ of a Banach algebra $\mathfrak{A}$, finitely generates the entire algebra $\mathfrak{A}$ just in case the set of all linear combinations of monomials of the form $f^{m} \bullet g^{n}$, where $m, n$ are natural numbers, is topologically dense in $\mathfrak{A}$. In particular, quantity algebras used in classical mechanics, thermodynamics, optics, and electromagnetism tend to be finitely generated. For example, in the formal treatment of measuring instruments, chosen to measure quantity $f$, we shall frequently rely on the pointer module's Banach algebra $\mathfrak{A}(\oslash f)$, generated by the instrument's pointer quantity $\oslash f$ and unit 1 . It is elementary to check that $\mathfrak{A}(\oslash f)$ is the smallest Banach algebra containing $\oslash f$. To see the measurementtheoretic significance of finitely generated quantity algebras, consider again the case of measuring quantity $f$ that results (say) in the outcome $c \in \operatorname{Val}(f)$. Since for any continuous function $\xi: \mathbb{R} \longrightarrow \mathbb{R}$ the value $\xi(c)$ can always be calculated (perhaps only approximately and of course likewise for all finitary combinations of the form $1+f+f \bullet f+f \bullet f \bullet f+\cdots$, etc.), there is no need to perform another measurement of measurand $\xi \circ f$. Simply, because measuring $f$ automatically takes care of the measurement of all quantities in the algebra $\mathfrak{A}(f)$ generated by $f$ (albeit only indirectly via computation), we can regard the measurement of $f$ as a physical process of extracting information from the target system about all quantities in the algebra $\mathfrak{A}(f)$. More generally, in the case of a joint measurement of quantities $f$ and $g$ the extracted information pertains to the finitely generated algebra $\mathfrak{A}(f, g)$, and so on.

Some (dimensionally homogeneous) quantities admit quantitative comparisons. A quantity $f \in \mathfrak{A}$ is said to be positive provided that it has the form $f=g^{2}=g \bullet g$ for some quantity $g$ in $\mathfrak{A}$ (e.g., the quantity area = length $\bullet$ length is positive). If $f$ is positive, then we shall write $f \geq O$, and $f \leq g$ is understood to mean $g-f \geq O$, where $O$ denotes the zero quantity with value space $\{0\}$. In this manner, $\mathfrak{A}$ is also furnished with a natural partial-order structure that is crucial in the statistical treatment of quantities. Positive quantities are particularly important in defining square roots and generally $n^{t h}$ roots of quantities of the form $\sqrt[n]{f}$. Specifically, the square root of a positive quantity $f$, denoted $\sqrt{f}$, is the unique positive quantity $g$ such that $g \bullet g=f$. The set of positive quantities of $\mathfrak{A}$ forms a linear lattice.

After a quantity algebra $\mathfrak{A}$ has been correctly chosen for the natural system under consideration, there is a general al- 
gebraic way to describe the system's possible (pure) deterministic and (mixed) probabilistic states in terms of suitable expectation functionals on $\mathfrak{A} .{ }^{1}$ In preparation for the correct conceptual setting for states, recall that the set of continuous linear functionals of the form $\mathscr{E}: \mathfrak{A} \longrightarrow \mathbb{R}$ is a Banach space (under pointwise-defined addition inherited from $\mathbb{R}$ and scalar multiplication), symbolized by $\mathfrak{A}^{*}$ and called the algebraic dual of Banach algebra $\mathfrak{A}^{2}$ An expectation functional $\mathscr{E}$ on $\mathfrak{A}$ is a unital, positive, continuous linear functional of the form above that ascribes a unique expected value $\mathscr{E}(f)$ to the quantity $f$ of interest, contigently upon the system's extant (deterministic or probabilistic) state, encoded by $\mathscr{E}$. We shall write

$$
\mathscr{S}(\mathfrak{A}){ }_{d f}\left\{\mathscr{E} \in \mathfrak{A}^{*} \mid \mathscr{E} \geq 0 \quad \& \quad \mathscr{E}(1)=1\right\}
$$

for the set all expectation functionals on $\mathfrak{A}$. Here the positivity constraint $\mathscr{E} \geq 0$ means $\mathscr{E}(f) \geq 0$ for all $f \geq O$ and, to reiterate, the number $\mathscr{E}(f)$ is thought to be the expected value of quantity $f$, when the target system is in a state represented by $\mathscr{E}$. Each expectation functional $\mathscr{E}$ is accompanied with its dispersion functional (algebraically emulating standard deviation), defined by $\operatorname{Dsp}_{\mathscr{E}}(f)={ }_{d f} \sqrt{\mathscr{E}\left(f^{2}\right)-\mathscr{E}(f)^{2}}$ for all quantities $f .^{3}$

Expectation functionals are needed for (i) extracting empirical content from the syntactic formalism of the quantity algebra $\mathfrak{A}$, (ii) determining the extant values of quantities during measurement, and for (iii) handling quantitative information about the target system in general. ${ }^{4}$ The so-called algebraic

\footnotetext{
${ }^{1}$ As will become increasingly evident as we proceed, a deterministic state of a target system at a given instant of time $t$ is the system's 'fine-scale mode of being' at $t$ as seen from the perspective of the quantity algebra $\mathfrak{A}$, brought about by its previous history, involving various preparations and causal interactions with other systems. States are essential in determining the extant values of quantities (using calculations or measurements) and in the reversed direction, the values of quantities at a given moment of time are collectively sufficient for individuating the system's prepared state at that time. In order to capture the supervening uncertainty or randomness inherent in repetitive measurement interactions and complex (e.g., chaotic) behaviors of systems, it is necessary to consider also a more general notion of state at any particular time $t$ (that prompts a substantially wider algebraic-analytic treatment of physical states), called a probabilistic state, intended to capture the system's 'gross mode of being' at $t$, i.e., its objective higher-level indefiniteness relative to $\mathfrak{A}$. Complete identification of a deterministic state comes with a maximum of information about exactly 'how the the target system is in itself' at a given time (when nobody looks), considered in relation to $\mathfrak{A}$. This information together with the system's governing laws is sufficient for specifying any state into which the system will evolve in the future. By contrast, in general, knowledge of a probabilistic state results only in a less-than-maximum information about the system.

${ }^{2} \mathrm{~A}$ Banach space is a linear space over $\mathbb{R}$ equipped with a norm $\|\cdot\|$ that is also a complete metric space under the metric induced by the norm. Banach spaces are the most important linear spaces arising in classical analysis. The so-called $L_{p}$ spaces are the prime examples of Banach spaces.

${ }^{3}$ To simplify our language, we shall often refer to an expectation functional somewhat loosely as a (deterministic or probabilistic) state, even though it is more precisely a mathematical representation of a physical state, relative to a selected quantity algebra, summarizing the target system's instantaneous situation or mode of being.

${ }^{4}$ The principal benefit of considering probabilistic states rather than just the customary deterministic states is that we immediately gain full access to a quantity-based algebraic probability calculus, needed for the treatment of
}

evaluation map

$$
\mathfrak{A} \times \mathscr{S}(\mathfrak{A}) \stackrel{€}{\longrightarrow} \mathbb{R}
$$

defined very simply by taking the expectation $€(f, \mathscr{E})={ }_{d f}$ $\mathscr{E}(f)$ of quantity $f$ when the system is in state $\mathscr{E}$, handles the algebraic specification of expectation values of quantities arising in calculations and measurements. A key observation we wish to make here is that each natural system $S$ of interest can be completely characterized by a quantity algebra $\mathfrak{A}_{\mathrm{S}}$ alone and there is a universal way to specify the algebra's state space $\mathscr{S}(\mathfrak{A})$ together with the evaluation map $€$. Note, however, that a theoretical account of the value $\mathscr{E}(f)$ does not automatically pop into a measuring instrument or into the experimenter's mind without a reproducible measurement process, based on a suitable physical interaction (i.e., exchange of energy and information) between the target system and a calibrated measurement apparatus for $f$. Referring to $\mathscr{E}(f)$ is of little value without having direct access to specific methods that actually generate, at the least, its rough estimate.

One may object that even if a measurement is instrumentbased or involves a physically correct measurement coupling, the outcome is still at best the expectation of $f$ and not the quantity's actual value. Here it is necessary to exercise care and not to jump to conclusions. As we shall see next, the outcome of measuring quantity $f$ decisively depends on the specific nature of the system's state encoded by a suitable expectation functional and on the physical nature of the quantity itself. For a simple example, if $f$ is two-valued, meaning $\operatorname{Val}(f)=\{0,1\}$, then $\mathscr{E}(f)$ specifies the probability that the actual value of quantity $f$ is 1 , given that the system is in the state $\mathscr{E}$. Other examples are provided below.

A distinguished role is played by the so-called extremal expectation functionals of $\mathscr{S}(\mathfrak{A})$, i.e., functionals that do not admit any proper convex decomposition. Formally, an expectation functional $\mathscr{E}$ is said to be extremal provided that $\mathscr{E}=$ $c \cdot \mathscr{E}_{1}+(1-c) \cdot \mathscr{E}_{2}$ implies $\mathscr{E}=\mathscr{E}_{1}=\mathscr{E}_{2}$ for all $0<c<1, \mathscr{E}_{1}$, and $\mathscr{E}_{2}$. Equivalently, a functional $\mathscr{E}$ is extremal just in case it is multiplicative, i.e., the equality $\mathscr{E}(f \bullet g)=\mathscr{E}(f) \cdot \mathscr{E}(g)$ holds for all quantities $f$ and $g$ in $\mathfrak{A}$. Extremal functionals $\mathscr{E}$ are precisely dispersion-free, i.e., we have the condition $\operatorname{Dsp}_{\mathscr{E}}(f)=0$ for all $f$. For future reference, the set of extremal expectation functionals is denoted by $\mathscr{S}_{\text {ex }}(\mathfrak{A}) .^{5}$ It turns out that its elements capture the so-called underlying deterministic states, involving minimal randomness and hence pro-

errors and uncertainties. We mention in passing that the pair $\langle\mathfrak{A}, \mathscr{E}\rangle$ consisting of a Banach algebra $\mathfrak{A}$ and an expectation functional $\mathscr{E}$ thereon can be thought of as a Banach algebra probability space that significantly generalizes Kolmogorov's classical notion of a probability space. For example, quantities $f$ and $g$ are said to be probabilistically independent relative to $\mathscr{E}$ provided that $\mathscr{E}(f \cdot g)=\mathscr{E}(f) \cdot \mathscr{E}(g)$. The algebraic analog of variance relative to $\mathscr{E}$ is defined by $\operatorname{Var}(f)=_{d f} \mathscr{E}\left((f-\mathscr{E}(f) \cdot 1)^{2}\right)$ for all quantities $f$. Many other probabilistic and statistical notions (e.g., covariance, correlation and conditional expectation) automatically carry over to Banach algebra probability spaces on an analogy with the corresponding concepts in classical probability and statistics.

${ }^{5}$ The value space of quantity $f$ can now be given quite simply by $\operatorname{Val}(f)=$ $\left\{\mathscr{E}(f) \mid \mathscr{E} \in \mathscr{S}_{\text {ex }}(\mathfrak{A})\right\}$. 
viding maximal information about the system's mode of being at a given moment of time. Since there is no dispersion when measuring a quantity $f$ in a deterministic state encoded by its extremal functional $\mathscr{E}$, the expectation $\mathscr{E}(f)$ determines the precise value of $f$. The objective of the remainder of this section is to clarify how and why mainline measurement problems pertaining to target systems can be framed in the setting of a suitable algebra $\mathfrak{A}$ of quantities (some of which are to be measured) and its associated compact convex subspace (topologically a simplex) $\mathscr{S}(\mathfrak{A}) \subset \mathfrak{A}^{*}$ of expectation functionals (serving as principal means of obtaining measurement outcomes), linked by an algebraic real-valued evaluation map $€$. We mention in passing that the simplex $\mathscr{S}(\mathfrak{A})$ of states comes with the lattice Face $(\mathscr{S}(\mathfrak{A}))$ of its faces. Recall that a face is an convex subset $\mathscr{F} \subseteq \mathscr{S}(\mathfrak{A})$ such that the condition $c \cdot \mathscr{E}+(1-c) \cdot \mathscr{E}^{\prime} \in \mathscr{F} \Longrightarrow \mathscr{E}, \mathscr{E}^{\prime} \in \mathscr{F}$ holds for all $0<c<1$. Two states $\mathscr{E}$ and $\mathscr{E} \prime$ in $\mathscr{S}(\mathfrak{A})$ are said to be orthogonal just in case the respective faces to which they belong are disjoint. As we shall see, the orthogonality relation between states and its cousins turn the lattice of faces into a rich playground for measurement theory. ${ }^{6}$ We now digress briefly to recall some crucial facts about mappings between state spaces and between their underlying quantity algebras, needed for a particularly attractive information-theoretic description of physical measurement.

Let $\mathscr{S}(\mathfrak{A})$ and $\mathscr{S}\left(\mathfrak{A}^{\prime}\right)$ be state spaces associated with Banach algebras $\mathfrak{A}$ and $\mathfrak{A}^{\prime}$, respectively, where in measurement applications the first algebra represents a target system and the second algebra models a measuring instrument or its pointer module. Then a map $\mathbf{C}^{*}: \mathscr{S}(\mathfrak{A}) \longrightarrow \mathscr{S}\left(\mathfrak{A}^{\prime}\right)$ between them is called a state channel or simply an information channel from input $\mathfrak{A}$ to output $\mathfrak{A}^{\prime}$ provided that the following three conditions are satisfied for all states $\mathscr{E}$ and $\mathscr{E} \prime$ on $\mathfrak{A}$, quantities $f$ in $\mathfrak{A}^{\prime}$, and reals $0 \leq c \leq 1$ :

(i) Affine Property: $\mathbf{C}^{*}\left(c \cdot \mathscr{E}+(1-c) \cdot \mathscr{E}^{\prime}\right)=c \cdot \mathbf{C}^{*}(\mathscr{E})+$ $(1-c) \cdot \mathbf{C}^{*}\left(\mathscr{E}^{\prime}\right)$.

(ii) Unity: $\left[\mathbf{C}^{*}(\mathscr{E})\right](1)=1$.

(iii) Positivity: $f \geq O \quad \Longrightarrow \quad\left[\mathbf{C}^{*}(\mathscr{E})\right](f) \geq 0$.

We call a state channel $\mathbf{C}^{*}: \mathscr{S}(\mathfrak{A}) \longrightarrow \mathscr{S}\left(\mathfrak{A}^{\prime}\right)$ deterministic just in case it maps deterministic states to deterministic states, encoded by extremal expectation functionals. ${ }^{7}$ It is easy to check that a cascade composition of two channels is also a channel. Additional properties of channels will be discussed later on. The measurement-theoretic significance of channels can be summed up as follows. Measurement is a physical operation performed on a target system, aimed at extracting information from the system that is transmitted via an information channel to the coupled measuring instrument's pointer

\footnotetext{
${ }^{6}$ We regard the quantities in $\mathfrak{A}$ to be instantiated in the target dynamical system collectively at all run times, while the states in $\mathscr{S}(\mathfrak{A})$ occur in the system only individually, one at a time.

${ }^{7}$ Strict determinism is usually reserved for bijective channels that preserve the orthogonality relation between states.
}

module for readout or storage. The input information is presumed to be encoded by an appropriate expectation functional that the system-to-instrument channel transforms during the measurement process into another closely related expectation functional at the receiving end, ready for decoding and interpretation. It is important to bear in mind that a channel captures not only the underlying causal mechanisms of the sender, receiver and transmitter, but also all physical (usually random) disturbances in the system - from source to destination - and randomness originating in the measurement process itself. In addition, we wish to emphasize that since channel transmission is inherently a finite process, in an actual realization each continuous input quantity will appear at the output in some discrete form.

In [13], Umegaki shows that each state channel $\mathbf{C}^{*}$ : $\mathscr{S}(\mathfrak{A}) \longrightarrow \mathscr{S}\left(\mathfrak{A}^{\prime}\right)$ defined above determines a unique dual channel, called a quantity channel or simply a cochannel, having the mathematical form $\mathbf{C}: \mathfrak{A}^{\prime} \longrightarrow \mathfrak{A}$ (with the arrow direction reversed and the superscript star symbol removed), such that the following conditions hold for all expectation functionals $\mathscr{E}$ on $\mathfrak{A}$, quantities $f, g$ in $\mathfrak{A}^{\prime}$, and reals $a, b \in \mathbb{R}$ :

(i) Duality: $\mathscr{E}(\mathbf{C}(f))=\left[\mathbf{C}^{*}(\mathscr{E})\right](f)$.

(ii) Linearity: $\mathbf{C}(a \cdot f+b \cdot g)=a \cdot \mathbf{C}(f)+b \cdot \mathbf{C}(g)$.

(iii) Unity: $\mathbf{C}(1)=1$.

(iv) Positivity: $f \geq O \quad \Longrightarrow \quad \mathbf{C}(f) \geq O$.

And conversely, each dual channel or simply cochannel (i.e., a linear unital positive map) $\mathbf{C}: \mathfrak{A}^{\prime} \longrightarrow \mathfrak{A}$ determines a unique (state) channel $\mathbf{C}^{*}: \mathscr{S}(\mathfrak{A}) \longrightarrow \mathscr{S}\left(\mathfrak{A}^{\prime}\right)$ such that the duality property listed above holds.

Following up on Umegaki's representation theorem, later we shall be discussing a general algebraic-analytic approach to a large variety of measurement processes, systematically framed in terms of the above defined channel-cochannel dual pair, succinctly symbolized by the map ${ }^{8}$

$$
\mathfrak{A}^{\prime} \times \mathscr{S}\left(\mathfrak{A}^{\prime}\right) \stackrel{\left(\mathbf{C}, \mathbf{C}^{*}\right)}{\longrightarrow} \mathfrak{A} \times \mathscr{S}(\mathfrak{A})
$$

rendering the adjointness diagram

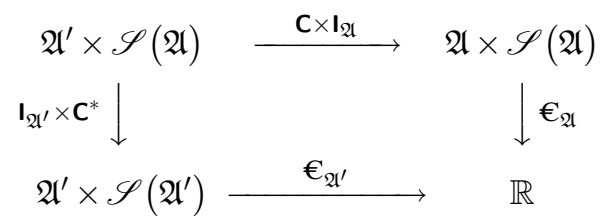

commutative. In this diagram, $\mathbf{I}_{\mathfrak{A}}$ denotes the identity mapping from $\mathscr{S}(\mathfrak{A})$ to itself and likewise $\mathbf{I}_{\mathfrak{A}^{\prime}}$ stands for the identity mapping of $\mathscr{S}\left(\mathfrak{A}^{\prime}\right)$ to itself. Finally, $€_{\mathfrak{A}}$ and $€_{\mathfrak{A}^{\prime}}$ are the basic evaluation maps, defined earlier. When unpacked, the commutativity property of the diagram simply states that the equality $\mathscr{E}(\mathbf{C}(f))=\left[\mathbf{C}^{*}(\mathscr{E})\right](f)$ holds for all states $\mathscr{E}$ and

\footnotetext{
${ }^{8}$ To indicate that the channel-cochannel pairs involve two maps, henceforth we shall denote them by triangle-headed arrows.
} 
quantities $f$. Because in what follows we shall need to refer to various quantity algebras, we prefer to reformulate the channel-cochannel duality in its above-displayed adjointness form. ${ }^{9}$ We take the two-way map $\left(\mathbf{C}, \mathbf{C}^{*}\right)$ (shown above) to be a fundamental concept in its own right that captures the information-theoretic essence of instrument-based measurement.

After these preliminaries we are now ready to define at least one major species (out of many possibilities) of a channeltheoretic measurement model as follows. Let $\mathfrak{A}_{\mathrm{S}}$ be the quantity algebra of a natural system $S$ under consideration and let $f \in \mathfrak{A}_{\mathrm{S}}$ be its measurand. Furthermore, take $\oslash f \in \mathfrak{A}_{\mathrm{M}}$ to be the measurand's pointer quantity in the measuring instrument's quantity algebra $\mathfrak{A}_{\mathbf{M}}$. Then a dual pair $\left(\mathbf{M}, \mathbf{M}^{*}\right)$, consisting of a linear unital positive map of the form $\mathbf{M}: \mathfrak{A}_{\mathrm{M}} \longrightarrow$ $\mathfrak{A}_{\mathrm{S}}$ and its associated affine unital map $\mathbf{M}^{*}: \mathscr{S}\left(\mathfrak{A}_{\mathrm{S}}\right) \longrightarrow$ $\mathscr{S}\left(\mathfrak{A}_{\mathrm{M}}\right)$ is said to represent an unbiased measurement of $f$ with pointer quantity $\oslash f$ provided that $\mathbf{M}(\oslash f)=f$. $\mathrm{A}$ closely related measurement model $\left(\mathbf{M}^{\prime}, \mathbf{M}^{\prime *}\right)$ with additive error is defined by $\mathbf{M}^{\prime}(\oslash f)=f+u$, where $u$ is an appropriate 'noise' quantity. Because the mathematical ways in which channels or cochannels are specified in applications tend to involve only one of them and the other is automatically derived from their duality property, often we shall refer to a cochannel (quantity channel) as the measurement model of interest, without explicitly specifying its corresponding (state) channel, or vice versa.

Up to this point we have been treating quantity algebras and their state spaces syntactically, i.e., in an axiomatic or presentation-independent way that treats all quantities on the same footing, intended to abstractly characterize and reason about the system's quantitative attributes of interest, with little regard for concrete numerical values presented in specific units, and methods or means of identifying these values. Likewise, states were modeled abstractly by positive normalized linear functionals on quantity algebras. The question thus arises: given the target system's quantity algebra and its accompanying state space, when can we say that they are 'correct' or meet our representational aims? Our basic problem is simply to find a method of quantity model validation. For that we need an interpretational framework, ${ }^{10}$ which secures a concrete physical meaning of quantities, their data propositions and laws - enabling a validation of propositions using measurement outcomes. Another way to express this is to say that in addition to the foregoing syntactic algebraic quantity and state apparatus, intended for effective theoretical analysis, we need their mathematically concrete realizations, implementations or distinguished representations, which allow us to designate a specific frame or basis, in which all pertinent physical variables and coordinates can be fixed for cal-

\footnotetext{
${ }^{9}$ We shall interpret channel $\mathbf{C}^{*}$ ontologically as a forward model of a measurement process that represents the causal direction of information flow from the target system to a measuring instrument. And its dual cochannel $\mathbf{C}$ is best thought of epistemically as an inverse model, representing the measurand's reconstruction or estimation operation, acting on its pointer quantity.

${ }^{10}$ Also known as a quantity algebra semantics, i.e., an endeavor of providing a mathematically specified empirical content for quantities and states.
}

culation, measurement, and testing.

This leads us to recall an important representation (realization) of Banach algebras. The so-called Gelfand representation theorem (see [6] and [11]) states that every abstractly given real (unital, commutative) Banach algebra $\mathfrak{A}$, satisfying the so-called $\mathbb{R}$-property $\|f\|^{2} \leq\left\|f^{2}+g^{2}\right\|$ for all quantities $f$ and $g$, is isomorphic to (and hence can be identified with) the concrete Banach algebra $\mathbf{C}(\mathscr{X})$ of all real-valued continuous functions (with the usual supremum norm) on a compact Hausdorff topological space $\mathscr{X}$, defined (among other options) by the space of maximal ideals in $\mathfrak{A}$ and furnished with the so-called Gelfand topology. ${ }^{11}$

The Gelfand duality between quantity algebras and topological state spaces, alluded to above, has proved to be surprisingly effective in the semantical study of measurement processes. As an additional element of concreteness, the socalled Riesz representation theorem (see [15] or Theorem 6.3 in [14]) states that the algebraic dual $\mathbf{C}(\mathscr{X})^{*}$ (formally a Banach space that includes all expectation functionals) of the mathematically concrete Banach algebra $\mathbf{C}(\mathscr{X})$ is isomorphic to (and hence can be identified with) the Banach space of all regular Borel measures on the Borel measurable space $\langle\mathscr{X}, \mathscr{F}\rangle$ associated with $\mathscr{X} .{ }^{12}$ And far more is mathematically true. The convex space $\mathscr{S}(\mathbf{C}(\mathscr{X}))$ of expectation functionals on the algebra $\mathbf{C}(\mathscr{X})$ is in a bijective correspondence with the space $\mathbf{P}(\mathscr{X})$ of all regular Borel probability measures on the induced Borel measurable space $\langle\mathscr{X}, \mathscr{F}\rangle$. Explicitly, each probability measure $P$ on the Borel state space $\langle\mathscr{X}, \mathscr{F}\rangle$ comes with its induced expectation functional $\mathscr{E}_{P}$ on $\mathbf{C}(\mathscr{X})$, defined by the integral $\mathscr{E}_{P}(f)={ }_{d f} \int_{\mathscr{X}} f(\mathrm{x}) P(\mathrm{dx})$ for all $f$ in $\mathbf{C}(\mathscr{X})$, and all expectation functionals arise in this way.

Conversely, it follows at once from Gelfand and Riesz representation theorems that for any underlying Banach algebra $\mathfrak{A}$ and a quantity $f$ in it, each state $\mathscr{E} \in \mathscr{S}(\mathfrak{A})$ determines a unique probability measure $P_{\mathscr{E}, f} \in \mathbf{P}(\operatorname{Val}(f))$ such that

$$
\mathscr{E}(\xi(f))=\int_{\operatorname{Val}(f)} \xi(\mathrm{x}) P_{\mathscr{E}, f}(\mathrm{dx})
$$

for all observables $\xi$ in $\mathbf{C}(\operatorname{Val}(f))$. Indeed, it is easy to see that since $\mathfrak{A}(f)$ is a subalgebra of $\mathfrak{A}$ and isomorphic to $\mathbf{C}(\operatorname{Val}(f))$, there is an embedding $f^{\sharp}: \mathbf{C}(\operatorname{Val}(f)) \longrightarrow \mathfrak{A}$, defined by the continuous function $f^{\sharp}(\xi)={ }_{d f} \xi(f)=\xi \circ \hat{f}$ for all $\xi$, where $\hat{f}$ Gelfand-represents $f$ along the lines discussed in the next paragraph. This representation result is convenient in formulating the notion of perfect measurement. By way of illustration, take $\mathfrak{A}_{\mathrm{S}}$ to be the quantity algebra of target system $\mathrm{S}$ and let $f \in \mathfrak{A}_{\mathrm{S}}$ be its measurand. Furthermore, take

\footnotetext{
${ }^{11}$ Here the term 'abstract' refers to those quantity algebras which are presented in a 'coordinate-free' manner - needed by the engineer or scientist to reason freely about the physical processes themselves, without invoking the unnecessary details of any specific numerical or other measurement-theoretic domains. However, the choice of these numerical domains is crucial in establishing a bridge between the world of numbers and the physical system's behavior, embodied in the representing quantity algebra and states thereon.

${ }^{12}$ Here the measure-theoretic Borel sigma-field $\mathscr{F}$ on $\mathscr{X}$ is generated by the open sets of the topological space $\mathscr{X}$.
} 
(7) $f \in \mathfrak{A}_{\mathrm{M}}$ to be the measurand's pointer quantity in the measuring instrument's quantity algebra $\mathfrak{A}_{\mathrm{M}}$. Then a cochannel (linear unital positive map) of the form $\mathbf{M}: \mathfrak{A}_{\mathrm{M}} \longrightarrow \mathfrak{A}_{\mathrm{S}}$ (together with its dual $\mathbf{M}^{*}$ ) is said to represent a perfect measurement of measurand $f$ with pointer quantity $\oslash f$ provided that $\operatorname{Val}(\oslash f)=\operatorname{Val}(f)$ and $\mathbf{M}(\xi(\oslash f))=\xi(f)$ for all continuous maps $\xi: \operatorname{Val}(f) \longrightarrow \mathbb{R}$. Returning to the general case, observe that the above-discussed passage

$$
\mathscr{S}(\mathfrak{A}) \stackrel{€_{f}^{*}}{\longrightarrow} \mathbf{P}(\operatorname{Val}(f)) \cong \mathscr{S}(\mathbf{C}(\operatorname{Val}(f)))
$$

from the abstractly given convex space of expectation functionals on $\mathfrak{A}$ to the concrete space of probability measures on the value space of a given measurand $f$, defined by $€_{f}(\mathscr{E})=$ $P_{\mathscr{E}, f}$, leads to an important commutative passage

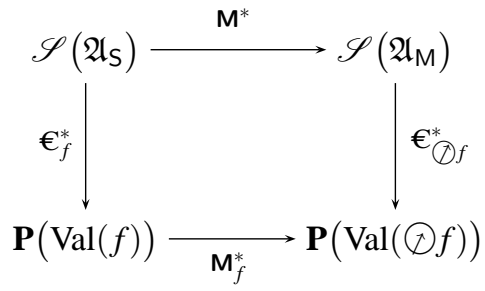

from a given (measurement) channel $\mathbf{M}^{*}$ to a Markov channel $\mathbf{M}_{f}^{*}$ between the measurand's value space input to its pointer quantity's value space output. In this way, a measurement channel can be thought of concretely as a transfer of input probability distributions on the measurand's values to suitable output probability distributions on its pointer quantity values. Such transfers are perfect if the distance between these two probability distributions is minimal.

Joint measurement of two measurands $f$ and $g$ is captured by the commutative diagram

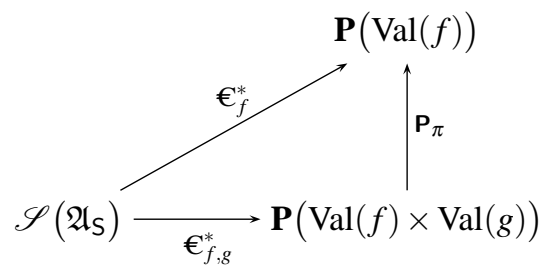

in which for each state $\mathscr{E}$ we set $€_{f, g}^{*}(\mathscr{E})=_{d f} P_{\mathscr{E}, f, g}$, so that for all continuous maps $\xi: \operatorname{Val}(f) \stackrel{\mathbb{R}}{\longrightarrow}$ we have

$$
\mathscr{E}(\xi(f))=\int_{\operatorname{Val}(f) \times \operatorname{Val}(g)} \xi(\mathrm{x}) P_{\mathscr{E}, f, g}(\mathrm{dx}, \mathrm{dy})
$$

and similarly for $\mathscr{E}(\zeta(g))$ with $\zeta: \operatorname{Val}(g) \longrightarrow \mathbb{R}$. Alternatively, if we rephrase the foregoing representation of joint measurement in a contravariant cochannel setting $€_{f, g}: \mathbf{C}(\operatorname{Val}(f) \times \operatorname{Val}(g)) \longrightarrow \mathfrak{A}_{\mathrm{S}}$, we obtain $€_{f, g}(\xi \otimes 1)=$ $\xi(f)$ and $€_{f, g}(1 \otimes \zeta)=\zeta(g)$.

Coming back to the representation of states, recall that the extremal functionals in $\mathscr{S}_{e x}(\mathbf{C}(\mathscr{X}))$ are in one-to-one and onto correspondence with the so-called Dirac probability measures on $\langle\mathscr{X}, \mathscr{F}\rangle$, i.e., probability measures of the form $D_{\times}$, concentrated at a single point $\mathrm{x}$ in $\mathscr{X}$ such that $\mathscr{E}_{D_{\mathrm{x}}}(f)=\hat{f}(\mathrm{x})$, where $\hat{f}: \mathscr{X} \longrightarrow \mathbb{R}$ (with $\operatorname{Val}(\hat{f})=\operatorname{Val}(f)$ and $\|\hat{f}\|=\|f\|)$ is the Gelfand representation of quantity $f$, specified by the chosen isomorphism ${ }^{\wedge}: \mathfrak{A} \longrightarrow \mathbf{C}(\mathscr{X})$. Because the set of Dirac probability measures is also in a bijective correspondence with the space $\mathscr{X}$ (i.e., we have the isomorphism $\left.\mathscr{S}_{e x}(\mathbf{C}(\mathscr{X})) \cong \mathscr{X}\right)$, the traditional state space terminology, usually reserved for space $\mathscr{X}$ alone, is consistent with the one introduced earlier. ${ }^{13}$ Considerations from Gelfand and Riesz representation results lead to the following mathematically concrete topological representation (realization) of the algebraic evaluation map $€$, introduced above:

$$
\mathbf{C}(\mathscr{X}) \times \mathbf{P}(\mathscr{X}) \stackrel{€_{\mathscr{X}}}{\longrightarrow} \mathbf{P}(\mathbb{R}),
$$

where for 'observable' $\hat{f}: \mathscr{X} \longrightarrow \mathbb{R}$ (Gelfand representing quantity $f$ ) in $\mathbf{C}(\mathscr{X})$ and a probability measure $P$ (encoding the system's extant probabilistic state) we set $€_{\mathscr{X}}(\hat{f}, P)=Q$ with $Q(B)={ }_{d f} P\left(\hat{f}^{-1}(B)\right)$ for all Borel subsets of $\mathbb{R}$. Thus, under a specific realization of the algebraic evaluation map $€$ the number $P\left(\hat{f}^{-1}(B)\right)$ gives the probability that upon (ideal) measurement the value of quantity $f$ will fall into the Borel subset $B$, given that the system is known to be in a state captured by the probability measure $P$. It is here that the quantity algebra actually makes contact with the natural system of interest. However, as we have already intimated, the expression $P\left(\hat{f}^{-1}(B)\right)$ provides only a theoretical determination of the value of quantity $f$, since it says nothing about exactly how the determination was made. For that we need a concrete measurement process which is described by a suitable tensor product of quantity algebras and its relations to the constituent quantity algebras, independently characterizing the target system and measuring instrument. The specifics will be discussed later on.

\footnotetext{
${ }^{13}$ Recall that in classical physics it is customary to attribute two kinds of states to any physical system under consideration: (i) the so-called groundlevel, pure, deterministic (maximally informative) states that are encoded by the points of a representing compact topological space $\mathscr{X}$ (or any of its isomorphic copies), and (ii) the logically higher-level probabilistic states, captured by probability measures in $\mathbf{P}(\mathscr{X})$. For example, from the standpoint of classical physics, a falling coin used in statisticians' coin-tossing experiments can certainly be viewed deterministically as a classical dynamical system with two distinguished terminal states, corresponding to the familiar heads and tails. However, since the solutions to the coin's equations of motion (not well known at the moment) are bound to be pathologically sensitive to initial conditions, a deterministic prediction of, say, the coin's landing with heads up, is not feasible in view of prohibitive limitations on the experimenter's memory, time, and other cognitive or physical resources, encountered in attempts at specifying the coin's initial conditions with perfect accuracy. Nonetheless, this macroscopic deterministic system exhibits in a structurally stable way also a (logically higher-level) unique probabilistic state, seen as an objective indefiniteness in its behavior, encoded by a probability measure, whose values are approximable by the frequencies of occurrence of heads. So, due to the extreme complexity of the coin's deterministic dynamics and states (challenging our resource-sensitive knowledge of the coin's precise initial state) and the inaccessible details regarding the coin's possible trajectories, it is incomparably more effective to work with the coin's probabilistic state than with its deterministic heads and tails. The fact that it is conceptually possible to eliminate the element of uncertainty lurking in a coin-tossing experiment is of little empirical significance, if in fact such uncertaintiy is not actually going to be removed. For more details regarding the stochastic aspects of scientific models, see [2] and [8].
} 
It should by now come as no surprise to the reader that when we analytically reason about, for example, the motion of a simple pendulum in a vertical plane, we may simply choose the real Banach algebra $\mathfrak{A}(\mathrm{p}, \mathrm{v})$, finitely generated by the position $\mathrm{p}$ of the pendulum's bob and its velocity $v$ (with respective value spaces $\operatorname{Val}(p)=\mathbb{C}$ and $\operatorname{Val}(v)=\mathbb{R})^{14}$ and frame all pertinent kinematic problems in it. However, if our task is to calculate or measure the value of the pendulum's position at a given time, we need to pass to the quantity algebra's concrete realization, namely the Banach algebra $\mathbf{C}(\mathbb{C} \times \mathbb{R})$ of all continuous real-valued functions on the state space $\mathbb{C} \times \mathbb{R}$ of all position-velocity pairs. Under this representation the position is now given by the first continuous projection map $\hat{\mathrm{p}}: \mathbb{C} \times \mathbb{R} \longrightarrow \mathbb{C} \subset \mathbb{R}$, where $\hat{\mathrm{p}}(a, b)=a$ for all position-velocity pairs $(a, b)$. And, as anticipated, the second projection $\hat{v}: \mathbb{C} \times \mathbb{R} \longrightarrow \mathbb{R}$ captures the pendulum's velocity quantity. A prime example of a secondary quantity in this representation is the all-important pendulum's total energy, i.e., the so-called Hamiltonian, specified in terms of $\hat{p}$ and $\hat{v}$. Under the foregoing quantity semantics, all probabilistic predictions of pendulum positions are readily handled by the evaluation map

$$
\mathbf{C}(\mathbb{C} \times \mathbb{R}) \times \mathbf{P}(\mathbb{C} \times \mathbb{R}) \stackrel{€_{\mathbb{R}}}{\longrightarrow} \mathbf{P}(\mathbb{R}),
$$

implemented by a suitable measurement process. Proceeding a stage further, it is important to realize that since these concrete mathematical models live in an infinitely resourced realm (where they can be subjected to infinitely precise mathematical operations), the (probability) values in $\mathbb{R}$ generally demand an infinite resource (e.g., unlimited time and memory for storage) to specify them precisely. In contrast, calculations and measurements are performed in a finite-resource framework, with strictly finite amounts of information. To bridge the gap between theoretical continuum dynamical models and finitary data structures, we need to construct appropriate converging families of discretized descent or offspring dynamical models of their continuum parent models. Details are given in Section 3 below.

\subsubsection{Von Neumann algebra approach to physical mea- surement}

In addition to real (unital, commutative) Banach algebras of continuous quantities, we shall also make frequent reference to von Neumann algebras $\mathfrak{N}$ of measurable (random) quantities, satisfying the customary algebraic closure condition

$$
f, g \in \mathfrak{N} \& c \in \mathbb{R} \quad \Longrightarrow \quad f+g, f \bullet g, c \cdot f \in \mathfrak{N},
$$

interpreted in a standard way. A real (unital, commutative) von Neumann algebra $\mathfrak{N}$ is a real (unital, commutative)

\footnotetext{
${ }^{14}$ We recall for the reader's convenience that $\mathbb{C}$ denotes the so-called circle group, defined by the half-open interval $[0,2 \pi)$ of reals - furnished with a cyclic group structure and topologically isomorphic to the unit circle that is obtained by identifying $2 \pi$ with 0 . The standard algebraic notation for the cyclic group $\mathbb{C}$ is $\mathbb{R} / 2 \pi \mathbb{Z}$.
}

normed algebra that is an algebraic dual (viewed as a Banach space) of a unique Banach space $\mathfrak{N}_{*}$, called the algebra's predual space, such that $\mathfrak{N}=\left(\mathfrak{N}_{*}\right)^{*}$. Although the language of von Neumann algebras closely parallels that of Banach algebras, there are several subtle formal and interpretational differences.

We have seen that in the world of Banach algebras quantities are the primary entities and (deterministic and probabilistic) states are treated via algebraic duality. In the universe of von Neumann algebras the quantity-state duality often proceeds in the reversed direction, in the sense that this time the quantity algebra is the unique dual of its presumed so-called predual Banach space, involving states. Remarkably, there is a measure-theoretic analog of the Gelfand representation result, known as the so-called spectral theorem (see [7], sections $9.3-9.5$ for a proof). It states that any abstractly given real (unital, commutative) von Neumann algebra $\mathfrak{N}$ with a normal state $\mathscr{E}$ thereon ${ }^{15}$ is isomorphic to (and hence can be identified with) the mathematically concrete von Neumann algebra $\mathfrak{L}_{\infty}(\mathscr{X}, \mathscr{F}, P)$ of bounded measurable real-valued functions (modulo $P$-probability measure zero) on a suitable classical probability space $\langle\mathscr{X}, \mathscr{F}, P\rangle$, where the essential supremum norm $\|\cdot\|_{\infty}$ of $\mathfrak{L}_{\infty}(\mathscr{X}, \mathscr{F}, P)$ is defined by

$$
\|f\|_{\infty}=\inf \{k>0|| f(\mathrm{x}) \mid \leq k \text { for } P \text {-almost all } \mathrm{x} \in \mathscr{X}\},
$$

and the algebra operations are defined pointwise, inherited from $\mathbb{R}$. Furthermore, under the chosen isomorphism ${ }^{\wedge}$ : $\mathfrak{N} \longrightarrow \mathfrak{L}_{\infty}(\mathscr{X}, \mathscr{F}, P)$ the expectation functional is given by $\mathscr{E}(f)=\int_{\mathscr{X}} \hat{f}(\mathrm{x}) P(\mathrm{dx})$ for all measurable quantities $f$ in $\mathfrak{N}$ with $\|f\|_{\infty}<\infty$. The spectral theorem provides a concrete mathematical semantics for abstractly conceived measurable (random) quantities and conversely, it shows how classical probability spaces are algebraically encodable by von Neumann algebras of quantities, equipped with normal states. (For technical details, see [12], Chapter 1, page 45.)

In addition to a concrete representation of measurable quantities, there is also a concrete representation of states. Specifically, the Banach space $\mathfrak{L}_{1}(\mathscr{X}, \mathscr{F}, P)=\mathfrak{L}_{\infty}(\mathscr{X}, \mathscr{F}, P)_{*}$ of all $P$-integrable functions on $\langle\mathscr{X}, \mathscr{F}, P\rangle$ is the predual of $\mathfrak{L}_{\infty}(\mathscr{X}, \mathscr{F}, P)$, so that we have $\mathfrak{L}_{1}(\mathscr{X}, \mathscr{F}, P)^{*}=\mathfrak{L}_{\infty}(\mathscr{X}, \mathscr{F}, P)$. Importantly, the subset $\mathscr{S}\left(\mathfrak{L}_{1}(\mathscr{X}, \mathscr{F}, P)\right)=_{d f}\left\{p \in \mathfrak{L}_{1}(\mathscr{X}, \mathscr{F}, P) \mid p \geq 0\right.$ \& $\|p\|=1\}$ of probabilistic states is actually the convex space of all density functions on $\langle\mathscr{X}, \mathscr{F}, P\rangle .^{16}$

Unlike Banach algebras (convenient for smooth and continuous quantities), von Neumann algebras tend to have many idempotent (two-valued, projection) quantities $f$ satisfying $f \bullet f=f$ (forming, roughly speaking, a Boolean sigma algebra under the partial ordering $\preccurlyeq$, defined by $f \preccurlyeq f^{\prime}$ if and

\footnotetext{
${ }^{15}$ A state $\mathscr{E}$ on $\mathfrak{N}$ is said to be normal provided that for every increasing sequence $f_{1}, f_{2}, \cdots$ of positive random quantities converging to $f$ in $\mathfrak{N}$ we have $\lim _{n} \mathscr{E}\left(f_{n}\right)=\mathscr{E}(f)$. Normalcy is a technical counterpart of the countable additivity property of probability measures.

${ }^{16}$ We mention in passing that $\mathfrak{L}_{\infty}(\mathscr{X}, \mathscr{F}, P)$ can also be obtained from the Banach algebra $\mathbf{C}(\mathscr{X})$ of continuous real-valued functions on $\mathscr{X}$ by completion under the so-called weak topology. For a detailed account of von Neumann algebras see [12].
} 
only iff $f \cdot f^{\prime}=f$ for all quantities $f$ and $f^{\prime}$ ), and are blessed with excellent convergence properties. For example, in the Banach algebra $\mathbf{C}([0,1])$ of continuous real-valued functions on the closed unit interval $[0,1]$ there are only two continuous idempotent (projection) quantities, namely, the constant quantities $\mathbb{k}_{0}, \mathbb{k}_{1}:[0,1] \longrightarrow \mathbb{R}$, defined by $\mathbb{k}_{0}(\mathrm{x})=0$ and $\mathbb{k}_{1}(\mathrm{x})=1$ for all $\mathrm{x}$, respectively. In sharp contrast, the characteristic function $1_{B}: \mathscr{X} \longrightarrow \mathbb{R}$ of each Borel measurable subset $B \subseteq \mathscr{X}$ of any compact Hausdorff space $\mathscr{X}$ is automatically an idempotent (two-valued) quantity in the von Neumann algebra $\mathfrak{L}_{\infty}(\mathscr{X}, \mathscr{F}, P)$, playing the role of events in the sense of Kolmogorov. In particular, the expected value $P\left(\hat{f}^{-1}(B)\right)=\mathscr{E}_{P}\left(1_{\hat{f}^{-1}(B)}\right)$ is the probability that the value of quantity $f$ lies in the real Borel set $B$, when the system is in a state encoded by $\mathscr{E}_{P} .{ }^{17}$ Since the algebra $\mathfrak{L}_{\infty}(\mathscr{X}, \mathscr{F}, P)$ contains only measurable functions, modulo probability measure $P$ zero, it can be generated by the set of characteristic functions $1_{B}$ of measurable subsets $B \subseteq \mathscr{X}$. (Any measurable quantity can be approximated arbitrarily well by a suitable linear combination of characteristic functions.)

It is time for an example. Along Newtonian lines, consider a quantity algebra for the kinematic behavior of a simple pendulum, swinging in a vertical plane. Earlier we mentioned that the correct quantity algebra for this target system is given by the Banach algebra $\mathbf{C}(\mathbb{C} \times \mathbb{R})$ continuous real-valued functions of the form $f: \mathbb{C} \times \mathbb{R} \longrightarrow \mathbb{R}$. The operations are defined pointwise and the norm of a quantity is the supremum of its absolute values. The points of $\mathbb{C} \times \mathbb{R}$ encode the pendulum's deterministic states (classically, in terms of its instantaneous position and velocity values), and each Borel probability measure $P$ thereon specifies a probabilistic state $\mathscr{E}_{P}$ in $\mathscr{S}(\mathbf{C}(\mathbb{C} \times \mathbb{R}))$ via the expectation integral $\mathscr{E}_{P}(f)=\int_{\mathbb{C} \times \mathbb{R}} f \mathrm{~d} P$ for all quantities $f$.

In a von Neumann algebra setting the pendulum's algebra of measurable quantities is given by the concrete von Neumann algebra $\mathfrak{L}_{\infty}(\mathbb{C} \times \mathbb{R}, \mathscr{F}, \Lambda)$ of bounded $\Lambda$-measurable real-valued functions on $\mathbb{C} \times \mathbb{R}$, where the relativizing default measure is the Lebesgue measure $\Lambda$. Here again, the operations are defined pointwise and the norm is the earlierintroduced essential supremum norm. The predual Banach space

$$
\mathfrak{L}_{1}(\mathscr{X}, \mathscr{F}, \Lambda)=\mathfrak{L}_{\infty}(\mathbb{C} \times \mathbb{R}, \mathscr{F}, \Lambda)_{*}
$$

of absolutely integrable functions contains all probability density functions on $\mathbb{C} \times \mathbb{R}$, comprising the convex subspace $\mathscr{S}\left(\mathfrak{L}_{1}(\mathscr{X}, \mathscr{F}, \Lambda)\right)$ of probabilistic states. Note that in this formalism the probabilistic states are conveniently encoded by probability density functions. And as above, the pendulum's position and velocity quantities are given by the measurable projections on the probability space $\langle\mathbb{C} \times \mathbb{R}, \mathscr{F}, \Lambda\rangle$.

Now we come to quantity algebras representing measuring instruments. Earlier we noted that in general it is not possible to obtain quantitative information about the target system's extant state without a physical interaction between the

\footnotetext{
${ }^{17}$ As common in probability theory, here as well as in what follows, the expression $1_{S}$ denotes the characteristic function (indicator) of the set $S$.
}

system and a measuring instrument, chosen for the measurand. In attempts at modeling the measurand's measuring instrument we assume that in addition to several constituents (e.g., sensor, transducer, and processors), the instrument has a pointer (or some other display) module that moves during the measurement process along a calibrated scale in such a way that different pointer positions on the scale correspond to different measurement outcomes. More particularly, there are two principal ways of modeling a measuring instrument. The first of these treats the instrument as an ordinary natural system, so that its characterizing quantities can be measured by other measuring instruments. Second, in the context of measurement involving the instrument under consideration we use a representation that focuses primarily on the instrument's pointer module, display of measurement results, and a statistical treatment of measurement errors.

For most practical purposes, then, even though a measuring instrument $\mathrm{M}(f)$ (designed to measure quantity $f$ ) comes with its own theoretical quantity algebra $\mathfrak{A}_{\mathrm{M}(f)}$ that completely characterizes the instrument's physical structure and behavior (important in explaining how the instrument works), for the sake of simplified analysis of a given measurement process we shall assume that the instrument's working quantity algebra $\mathfrak{A}(\oslash f)$ is specified more modestly by the measurand's pointer (output) quantity $\oslash f$, whose values (thanks to calibration) provide quantitative information about the objective values of measurand $f$. Abstractly, the instrument's pointer (e.g., a needle) can be thought of as a particle moving continuously in one dimension only (e.g., on a half circle) and its other degrees of freedom are simply ignored. This is a theoretical view of classical measuring instruments. However, due to the instrument's limited sensitivity, discrimination, accuracy, finitary resources, and external noise, the actual position information provided by the pointer quantity $\oslash f$ about its measurand $f$ is bound to be only partial and unreliable in general. Characteristically, normal measurement outcomes are small intervals containing the pointer quantity's values. For example, suppose the experimenter reads the dial (or digital display) of the measuring instrument $\mathrm{M}(f)$ and reports that the value of $\oslash f$ (in some given units) is 3.450. What this means is that the pointer quantity's actual value lies in the interval [3.4495,3.4505], providing only an estimate of the objective value of measurand $f$. In Section 3 we shall be discussing several related problems from the standpoint of discretization of quantities and their algebras.

Having introduced the quantity algebras for measuring instruments we will be using later on, we now briefly mention the formal description of their (deterministic and probabilistic) states. In the case of continuous quantities, we may set the model of the instrument's pointer module (for measurand $f)$ to be the Banach algebra $\mathbf{C}(\operatorname{Val}(\oslash f))$ of (bounded) continuous real-valued functions on the value space $\operatorname{Val}(\oslash f)$, viewed as the pointer's underlying state space. In this way, we have access not only to the measurand's internal pointer quantity $l_{f}: \operatorname{Val}(\oslash f) \longrightarrow \mathbb{R}$ (defined by the subspace inclusion $l_{f}(\mathrm{x})=\mathrm{x}$ for all $\mathrm{x}$ ), but also to all continuous functions 
defined on its set $\operatorname{Val}(\oslash f)$ of measurement outcomes. As anticipated, probabilistic states are encoded by expectation functionals in $\mathscr{S}(\mathbf{C}(\operatorname{Val}(\oslash f))) \cdot{ }^{18}$

In the ambience of measurable quantities, the instrument's pointer module for measurand $f$ is conveniently modeled by the von Neumann algebra $\mathfrak{L}_{\infty}(\operatorname{Val}(\oslash f), \mathscr{F}, \Lambda)$ of all bounded measurable real-valued functions on the pointer quantity's value space $\operatorname{Val}(\oslash f)$, equipped with the Lebesgue measure. As we have already indicated, probabilistic states are usually encoded by density functions on $\operatorname{Val}(\oslash f)$. We break off our discussion of quantity algebras and their states, since we have gone far enough to show how they work. We now turn to the discussion of (prediction and measurement) data lattices of quantities.

\subsection{Data lattices of quantity algebras}

In this subsection we embark on a brief study of quantities in terms of their data lattices. Although this is not always made fully explicit in the literature on classical measurement theory, in parallel with the assignment of a minimal quantity algebra $\mathfrak{A}_{\mathrm{S}}$ to a target system $\mathrm{S}$ there is also an association of a data lattice - symbolized by $\mathfrak{L}_{\mathrm{S}}$, intended for expressing various claims about quantities possessed by $S$ in terms of their values ${ }^{19}$ and designed for reasoning about the target system's instantaneous continuous or measurable features and estimates thereof. Data lattices come in three basic flavors: (i) equational, (ii) comparative, and (iii) probabilistic.

In the simplest deterministic and most idealized situation, there is a consideration of an elementary equational evaluation mapping $\bumpeq: \mathfrak{A}_{\mathrm{S}} \times \mathbb{R} \longrightarrow \mathfrak{L}_{\mathrm{S}}$ that assigns to each quantitymagnitude pair $(f, c)$ a unique elementary proposition (written in an infix form) $f \bumpeq c .^{20}$ Its physical meaning under the classical realist interpretation is as follows: Quantity $f$ has an objective value and that value is equal to $c$ at a given instant of time. ${ }^{21}$ We mention in passing that reasoning about quan-

\footnotetext{
${ }^{18} \mathrm{Here}$ we would like to emphasize again that in general these so-called theoretical models of quantities and states assume that the underlying domains are continua (i.e., they are locally homeomorphic to the real line). Yet all measurement outcomes are known to be relatively small discrete rational numbers or histograms constructed from simple relative frequencies. The recurring point is that for reasons of effective mathematical tractability, theoretical models are bound to be far more idealized than warranted by the actual resource-sensitive physical situation they purport to represent.

${ }^{19}$ Remember that quantities are assumed to be instantiated by the target system in distinguishable degrees or amounts, expressible by numbers in some units, comprising their value space.

${ }^{20}$ Since proposition $f \bumpeq c$ is logically equivalent to $f-c \cdot 1 \bumpeq 0$, formally it is sufficient to consider only equational propositions of the form $f \bumpeq 0$, satisfying the equivalence $(f \bumpeq 0 \vee g \bumpeq 0) \Longleftrightarrow f \bullet g \bumpeq 0$ for all quantities $f$ and $g$.

${ }^{21}$ Here we wish to emphasize that in classical physics the realist position is that quantities possess their values independently of whether or not they are measured. Furthermore, quantities possess their empirical meaning independently of the measurement methods which may be available for them. In contrast, some empiricists interpret propositions of the form $f \bumpeq c$ in a considerably weaker counterfactual manner thusly: If quantity $f$ were measured by a designated measuring instrument $\mathrm{M}(f)$, then the measurement result would be $c$. For us, if $f$ were measured by a designated instrument, then in general the measurement result provided by the pointer quantity $\oslash f$ of $f$ would only
}

tities and their values in the data lattice $\mathfrak{L}_{\mathrm{S}}$ requires several logical and algebraic rules that - bearing in mind space limitations - we do not list. Although we shall continue to work in a Banach algebra framework, obviously, the assignment of data propositions to quantity algebras works equally well also in the context of von Neumann algebras.

At this point we are interested in knowing when is an elementary data proposition of the form $f \bumpeq c$ true? In classical measurement such data propositions (serving as units of predictive or measurement information) and their logical combinations describe certain instantaneous features of the target system that are either possessed or not possessed by the system, depending on how the system is, independently of any measurement or experimenter. In this way, the system's (deterministic) state becomes essential in specifying whether or not the foregoing data proposition is true. Explicitly, the state space $\mathscr{X}_{S}$ of the quantity algebra $\mathfrak{A}_{\mathrm{S}}$ (determined uniquely by the Gelfand representation to within a homeomorphism) provides an effective realist semantics for all propositions about quantities in the associated data lattice $\mathfrak{L}_{\mathrm{S}}$. It is easily established that each deterministic state $\mathrm{x} \in \mathscr{X}_{\mathrm{S}}$ assigns to proposition ' $f \bumpeq c$ ' a unique truth value. Concretely, proposition ' $f \bumpeq c$ ' is said to be true about quantity $f$ (and the content of what it signifies is actualized by the target system) in state $\mathrm{x}$ exactly when $\hat{f}(\mathrm{x})=c$, where $\hat{f}: \mathscr{X} \longrightarrow \mathbb{R}$ is the Gelfand 'observable' representing $f$. Evidently, the truth value of a complex data proposition is a logical combination of truth values of its simple constituents. Formally, the topological statespace semantics of proposition $f \bumpeq c$ is given by the (closed or measurable) subset

$$
\llbracket f \bumpeq c \rrbracket=_{d f}\{\mathrm{x} \in \mathscr{X} \mid \hat{f}(\mathrm{x})=c\}
$$

of those states in which the representing 'observable' of quantity $f$ takes the value $c$. Thus, we have specified a semantic valuation map

$$
\llbracket \cdot \rrbracket: \mathfrak{L}_{\mathrm{S}} \longrightarrow \operatorname{Sub} \mathscr{X}_{\mathrm{S}}
$$

from the target system's data lattice to the concrete lattice of subsets of $\mathscr{X}_{\mathrm{S}}$. Here the technical details depend on whether $\hat{f}$ is assumed to be continuous or measurable. For example, in the von Neumann algebra framework of measurable quantities, the logic of $\mathfrak{L}_{\mathrm{S}}$ is Boolean. However, since in the case of continuous quantities the set $\llbracket f \bumpeq c \rrbracket=\{\mathrm{x} \in \mathscr{X} \mid \hat{f}(\mathrm{x})=c\}$ is a closed subset of the compact Hausdorff topological space $\mathscr{X}_{\mathrm{S}}$, the resulting lattice is Brouwerian. In general, data lattices mirror the underlying geometric structure of their state spaces. Because in a von Neumann algebra setting the characteristic function $1_{\llbracket f \bumpeq c \rrbracket}$ is automatically a two-valued (idempotent) measurable quantity (with $1_{\llbracket f \bumpeq c \rrbracket}(\mathrm{x})=1$ if $\hat{f}(\mathrm{x})=c$, and 0 otherwise), the expectation $\mathscr{E}\left(1_{\llbracket f \bumpeq c \rrbracket}\right)$ gives the proba-

be an estimate or an approximation of $c$. These two diametrically opposing interpretations are particularly significant in the theory of quantum measurement. In general, the actual value of a quantity at a given time - assumed to exist from the perspective of $\mathfrak{A}_{\mathrm{S}}$ - cannot be known exactly. Quantum effects may even prevent the existence of such a value. However, various approximations of this idealized value are presumed to be known or knowable. 
bility $P_{\mathscr{E}}(f \bumpeq c)$ that quantity $f$ has the value $c$, given that the system is in a state encoded by $\mathscr{E}^{22}$

One major disadvantage of equational data propositions is that in view of various resource limitations, in general experimenters cannot know the exact values of quantities. However, in the case of comparative propositions of the form $f \lessdot c-$ expressing the fact that quantity $f \in \mathfrak{A}_{\mathrm{S}}$ has a value and that value is strictly smaller than $c$ - things are rather better. In particular, compound data propositions of the form $a \lessdot f \lessdot b$ lead to an important interval calculus for the treatment of quantity values. Since the state-space semantics proceeds on the same line of reasoning as in the case of equational propositions, it seems unnecessary to go through the details. Measurement outcomes encoded by data propositions of the form $\oslash f \bumpeq c$ provide complete information about the value of measurand $f$ at a given time. However, most measurements extract only partial information about the measurand, discussed below.

A considerably more general and less idealized class of propositions is based on a membership mapping $\Subset: \mathfrak{A}_{\mathrm{S}} \times$ $\mathscr{B}_{\mathbb{R}} \longrightarrow \mathfrak{L}_{\mathrm{S}}^{\prime}$ that assigns to each quantity $f$ and a Borel measurable subset $B$ of the real line $\mathbb{R}$ the proposition $f \notin B$, written once again in an infix form. Its intended physical meaning under the realist interpretation is the following: Quantity $f$ has an objective value and that value lies in the real Borel subset $B .{ }^{23}$ Reasoning about quantities and their values in the extended Borel data lattice $\mathscr{L}_{\mathrm{S}}^{\prime}$ requires additional logical and algebraic rules we do not list. Regarding semantics, a proposition ' $f \in B$ ' is said to be true about quantity $f$ (and its corresponding state of affairs is realized in the target system) in state $\mathrm{x} \in \mathscr{X}$ provided that $\hat{f}(\mathrm{x}) \in B$. In general, the state-space semantics of a data proposition $f \in B$ is given by the subset

$$
\llbracket f \Subset B \rrbracket=_{d f}\{\mathrm{x} \in \mathscr{X} \mid \hat{f}(\mathrm{x}) \in B\}=\hat{f}^{-1}(B)
$$

of those states in which the associated observable of quantity $f$ takes values belonging to $B$. Thus, we have now specified a new semantical valuation map $\llbracket \cdot \rrbracket: \mathfrak{L}_{S}^{\prime} \longrightarrow$ Sub $\mathscr{X}_{S}$ from the target system's extended data lattice to the concrete Borel or Brouwer algebra of subsets of $\mathscr{X}_{\mathrm{s}}$.

Since physical measurements are regularly subjected to uncertainties and randomness, results of measurement are frequently represented in terms of probabilistic propositions of the form $\mathscr{E}\left(1_{\llbracket f \notin B \rrbracket}\right)=p$ or $P_{\mathscr{E}}(f \notin B)=p$, stating that the value of quantity $f$ lies in the real Borel subset $B$ with probability $p$, given that the system is in state $\mathscr{E}$. As we have seen, the probability measure $P_{\mathscr{E}}$ on $\mathscr{X}$ is obtained by a concrete representation of the expectation functional $\mathscr{E}$ in $\mathscr{S}\left(\mathfrak{A}_{\mathrm{S}}\right)$, using Riesz representation result.

We now know that each quantity algebra $\mathfrak{A}$ comes with its associated (equational or Borel) data lattice $\mathfrak{L}_{\mathfrak{A}}$, endowed

\footnotetext{
${ }^{22}$ We mention in passing that we also have the algebraic semantics of propositions, given by the subset $\llbracket f \bumpeq c \rrbracket_{\text {alg }}=_{d f}\left\{\mathscr{E} \in \mathscr{S}_{e x}(\mathfrak{A}) \mid \mathscr{E}(f)=c\right\}$ of extremal functionals. However, in view of Gelfand representation, the difference between the algebraic and topological semantics is largely conceptual.

${ }^{23}$ In applications, instead of using arbitrary Borel subsets, it is sufficient to use only open (or closed) intervals with rational end-points.
}

with a convenient logic to reason about quantity values. ${ }^{24}$ An equational data lattice of propositions represents the target system's actual or potential instantaneous measurable features. The association of equational propositions with quantities is illustrated in Figure 1 below:

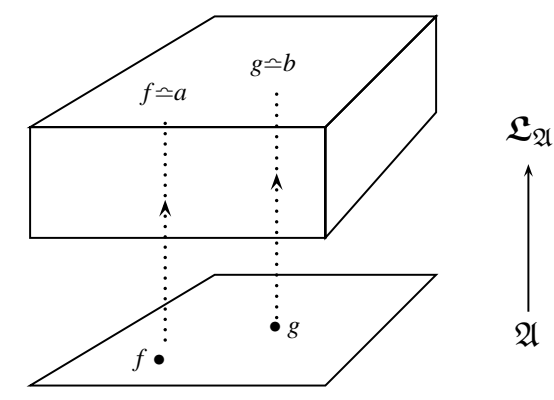

Fig. 1 Data lattice of quantity algebras

Before leaving this subsection, we make a quick remark about the relevance of data lattices in von Neumann algebra settings. Because each proposition of the form $a \lessdot f \lessdot b$ (and its Borel subset generalizations involving a measurable quantity $f$ ) canonically transforms into a two-valued quantity $1_{\llbracket a<f<b \rrbracket}$ that receives value 1 just in case the value of $f$ lies strictly between the reals $a<b$, and 0 otherwise, we can also think of the indicator $1: \mathfrak{L}_{\mathrm{S}}^{\prime} \longrightarrow \mathfrak{N}_{\mathrm{S}}$ as an embedding function that maps data propositions to two-valued (idempotent, projection) quantities of the von Neumann algebra $\mathfrak{N}_{S}$. In particular, $\mathscr{E}\left(1_{\llbracket a<f<b \rrbracket}\right)$ is equal to the probability that the objective value of quantity $f$ lies in the open interval $(a, b)$, given that the system is in state $\mathscr{E}$. Seen in this light, the intimate relationship between quantity algebras and data lattices goes even deeper. By analogy with channels and cochannels between von Neumann (Banach) algebras, there are Boolean (Brouwer) channels between data lattices, traceable to the two-valued evaluation map $€: \mathfrak{L}_{\mathrm{S}} \times \mathscr{X}_{\mathrm{S}} \longrightarrow\{0,1\}$, defined by $€(f \bumpeq c, x)=1$ if $\hat{f}(\mathrm{x})=c$ and 0 otherwise.

\subsection{An example of a static length measurement with error}

We now have at our disposal several concepts and methods of characterizing simple measurement processes and their outcomes in algebraic settings. In this subsection we present an elementary example of a classical, comparison-based, static measurement of length, involving deterministic (systematic) and probabilistic (random) measurement error.

Suppose we want to measure the length of a flagpole (or that of a medium-size straight rod, and so forth), using a yardstick or a tape measure, marked with carefully calibrated uniform subdivisions in inches, centimeters, or in some other

\footnotetext{
${ }^{24}$ As usual in logic, the meet operation in the data lattice corresponds to conjunction and the join operation refers to disjunction of propositions. It is not difficult to see that the false sentence $1 \bumpeq 0$ specifies the bottom element $\perp$ and the true sentence $1 \bumpeq 1$ determines the top element $T$ of the equational data lattice. It is well known that in classical physics data lattices carry the structure of a Boolean algebra or that of a Brouwer algebra, whereas in quantum physics the lattice structure is only weakly modular, orthocomplemented, and decisively nondistributive.
} 
units of the length dimension. Obviously, the target natural system instantiates several geometric, thermodynamical and other quantities, including length, diameter, temperature, and so on, but we shall focus only on the flagpole's length quantity $\ell$ with its value space defined (for concreteness) by $\operatorname{Val}(\ell)=[0, L] .{ }^{25}$ Continuing in the spirit of the previous subsections, it may be natural to assume that in view of continuity being an important regulative principle for reasoning about length, flagpole length measurements should be modeled by the Banach algebra $\mathfrak{A}(\ell) \cong \mathbf{C}(\operatorname{Val}(\ell))$ generated by $\ell$, and we may want to proceed similarly in modeling the yardstick. This approach is adequate in the context of static deterministic measurement. However, since any mathematical model of length measurement with random error and uncertainty in the measurement outcomes will have to account for the inherent element of randomness in order to be adequate, we find it more appropriate to represent the target system by the von Neumann algebra $\mathfrak{N}(\ell)$, generated by quantity $\ell$ and Rieszisomorphic to a concrete algebra $\mathfrak{L}_{\infty}([0, L], \mathscr{F}, P)$, where $\ell$ is now regarded as a real-valued random variable with a probability distribution $P .^{26}$ It should be noted that in measuring length $\ell$, the quantity algebra $\mathfrak{N}(\ell)$ automatically extends its measurement results also to squares $\ell \bullet \ell$ (built from flagpoles), cubes $\ell \cdot \ell \bullet \ell$, and a host of other systems that instantiate quantities definable in terms of $\ell$. In a deterministic situation it is standard to assume that the flagpole has a definite (albeit unknown) length, say $\ell \bumpeq \lambda$, and therefore its state (relative to the representing algebra $\mathfrak{N}(\ell) \cong \mathfrak{L}_{\infty}([0, L], \mathscr{F}, P)$ ) is captured by the maximally informative Dirac probability measure $D_{\lambda}$ on $[0, L]$, giving $D_{\lambda}(\llbracket \ell \bumpeq c \rrbracket)=1$, if $c=\lambda$, and 0 otherwise. However, in view of our interest in measurement error, we wish to consider epistemically less idealized situations in which the flagpole's actual length is described nondeterministically by a probabilistic state that is different from Dirac probability distributions.

In measuring the flagpole's length using a yardstick, generally it is impossible to determine the exact length value for at least two simple reasons: (i) the scale of the yardstick is known to have a limited resolution and accuracy, specified by finitely spaced marks and less-than superbly calibrated subdivision, and (ii) stepping off the yardstick against the flagpole usually involves small length disparities in its imperfect placements alongside the flagpole, misjudgments of tiny fractions of length on the scale, and parallax errors in outcome

\footnotetext{
${ }^{25}$ The upper bound $L$ of the closed real interval $[0, L]$ of possible length values can be set to exceed the lengths of all possible real-world flagpoles or it can simply be the Hubble length, specified by the size of the observable universe.

${ }^{26}$ This algebraic model seems reasonable, since at any given time the flagpole's actual length is subtly determined also by fluctuating temperature, pressure and other changes in the flagpole's material composition and its environment - not to mention randomness stemming from the measurement procedure itself. We mention in passing that our treatment of length measurement carries over, with only notational changes, also to mass measurement on a chemical balance with a calibrated pointer system, volume measurement of liquids using a graduated measuring cup, and so forth. And of course, the measurement model is meant to apply to any and therefore all flagpoles or rods.
}

reading. We cast all this in the framework of an associated random pointer quantity $\ell_{\varepsilon}$ with its finite value space defined (for example) by $\operatorname{Val}\left(\ell_{\varepsilon}\right)=\varepsilon \mathbb{N} \cap[0, L]$, where the set $\varepsilon \mathbb{N}=\{0, \varepsilon, 2 \varepsilon, \cdots\}$ is comprised of integer multiples of a fixed unit length $\varepsilon$, say, one inch, chosen by the measurer. Intuitively, the yardstick-based measurement process will round off the flagpole's actual length to that integer multiple of the chosen unit which is closest to it. Naturally, yardsticks marked off in smaller length units $\varepsilon$ will provide appropriately more accurate measurement results, but of course they will not be brought into a one-to-one correspondence with the possible lengths in $[0, L]$.

For future technical needs, let $\lceil\lambda\rceil$ be the integer part of real number $\lambda$, so that we have $\lambda-1<\lceil\lambda\rceil \leq \lambda$ and $\lambda-\lceil\lambda\rceil$ is the fractional part of $\lambda$. We now have at our disposal a natural (generally nonlinear) projective round-off map $\Re_{\varepsilon}:[0, L] \longrightarrow[0, L]_{\varepsilon}$, defined by $\Re_{\varepsilon}(\lambda)={ }_{d f}\left[\frac{\lambda}{\varepsilon}+\frac{1}{2}\right] \cdot \varepsilon$, that will play a crucial role in our study of discretization in Section 3 . The term is aptly chosen, since $\mathfrak{R}_{\varepsilon}$ literally rounds off a given length (specified by a real number) to the integer multiple of $\varepsilon$ that is numerically closest to it. We also have an accompanying embedding map $\mathfrak{I}_{\varepsilon}:[0, L]_{\varepsilon} \longrightarrow[0, L]$ such that $\left[\Re_{\varepsilon} \circ \mathfrak{I}_{\varepsilon}\right](k \cdot \varepsilon)=\mathfrak{R}_{\varepsilon}(k \cdot \varepsilon)=k \cdot \varepsilon$ and $\left[\mathfrak{I}_{\varepsilon} \circ \Re_{\varepsilon}\right](\lambda) \leq \lambda+\frac{1}{2}$ for all $\lambda$. Finally, note that $\Re_{\varepsilon}\left(2 k \cdot \varepsilon+\frac{1}{2}\right)=(k+1) \cdot \varepsilon$.

Just as in the case of the flagpole's length quantity, so too it is appropriate to model the measuring instrument and associated comparison-based measurement process by the von Neumann algebra $\mathfrak{N}\left(\ell_{\varepsilon}\right)$, generated by $\ell_{\varepsilon}$ and Riesz-isomorphic to $\left.\mathfrak{L}_{\infty}\left([0, L]_{\varepsilon}\right), \mathscr{F}_{\varepsilon}, P_{\varepsilon}\right)$, where $P_{\varepsilon}$ is reserved for a probability distribution of error-laden measurement outcomes that is estimated from a sequence of independently repeated measurements.

From our brief remarks above it follows that in the most common deterministic case, length measurement with a yardstick will display the round-off value (i.e., the nearest integer multiple of the chosen measurement unit $\varepsilon>0) \Re_{\varepsilon}(\lambda)$, given that the flagpole's actual length is $\lambda$. In the language of data propositions this can be summarized by the conditional

$$
\ell \bumpeq \lambda \quad \Longrightarrow \quad \ell_{\varepsilon} \bumpeq \Re_{\varepsilon}(\lambda) .
$$

We have already emphasized that states (encoded by expectation functionals) are essential in determining the values of quantities. Accordingly, a deterministic measurement of the flagpole's length quantity $\ell$ with the yardstick's pointer quantity $\ell_{\varepsilon}$ is specified by a projective deterministic state channel

$$
\mathscr{S}\left(\mathfrak{L}_{\infty}([0, L], \mathscr{F}, P)\right) \stackrel{\mathbf{M}^{*}}{\longrightarrow} \mathscr{S}\left(\mathfrak{L}_{\infty}\left([0, L]_{\varepsilon}, \mathscr{F}_{\varepsilon}, P_{\varepsilon}\right)\right),
$$

satisfying $\mathbf{M}^{*}\left(\mathscr{E}_{D_{\lambda}}\right)=\mathscr{E}_{D_{\mathfrak{R}_{\varepsilon}(\lambda)}}$ for all $\lambda$ in $[0, L]$, where (following Riesz representation) $\mathbf{M}^{*}\left(\mathscr{E}_{D_{\lambda}}\right)$ is the extremal linear functional specified by the Dirac probability measure $D_{\lambda}$. For all practical purposes the foregoing state channel abstractly captures a trivial fact, namely, that in measuring the length of a flagpole using a yardstick, we shall generally obtain an outcome that is only approximately equal to the flagpole's actual length, with an error less than $\varepsilon$ units. This implies, again 
trivially, that in view of the yardstick's limited accuracy, static deterministic measurement will classify different flagpoles as equally long and hence epistemically equivalent, given that their round-off values are detected as equal. This underdetermination of the flagpole's real length is encoded by the state channel's projective (onto) property.

Now, if we change our point of view somewhat and switch to the dual quantity channel (cochannel) representation, specified by the deterministic measurement model

$$
\mathfrak{L}_{\infty}\left([0, L]_{\varepsilon}, \mathscr{F}_{\varepsilon}, P_{\varepsilon}\right) \stackrel{\mathrm{M}}{\longrightarrow} \mathfrak{L}_{\infty}([0, L], \mathscr{F}, P),
$$

where $\mathbf{M}$ is now a von Neumann algebra embedding, obtained from $\Re_{\varepsilon}$ and specified by the step function $\mathbf{M}\left(\ell_{\varepsilon}\right)=\widehat{\ell}$ with $\widehat{\ell}(\lambda)=k \cdot \varepsilon$, if $\frac{\varepsilon}{2} \cdot(2 k-1) \leq \lambda<\frac{\varepsilon}{2} \cdot(2 k+1)$ for all $k \geq 1$, and $\widehat{\ell}(\lambda)=0$ for $\lambda<\frac{1}{2}$, we obtain a recipe for the reconstruction of $\ell$ in terms of $\widehat{\ell}$ from measurement data. The graphs of $\ell$ and $\widehat{\ell}$ are given in Figure 2 below.

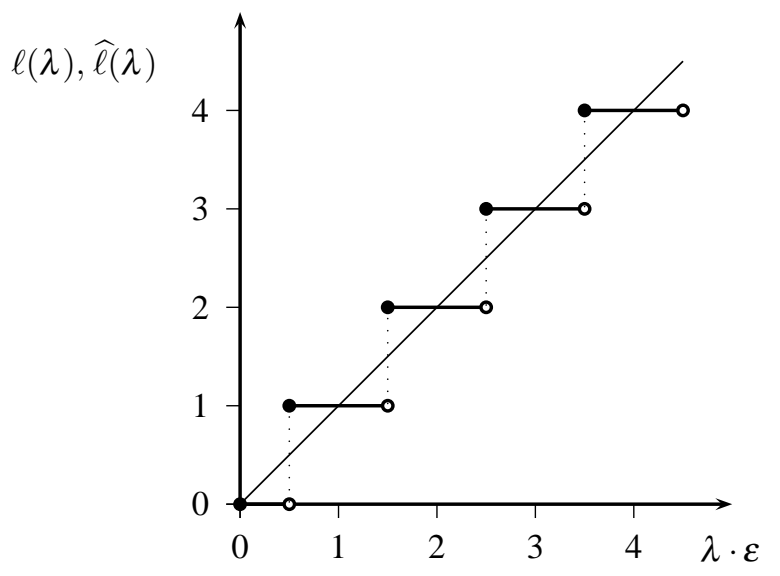

Fig. 2 Step function approximation of the actual length quantity

We see that $\widehat{\ell}$ is the best estimator for the flagpole's length quantity $\ell$, based on the measuring instrument's pointer quantity $\ell_{\varepsilon}$. In addition, note that $\widehat{\ell}$ is discontinuous at points with maximal error. However, in view of the measurement error (maximal bias) specified by $\left\|\mathbf{M}\left(\ell_{\varepsilon}\right)-\ell\right\|=\frac{1}{2} \varepsilon$, the measurement model under consideration is not able to reconstruct $\ell$ from its pointer quantity $\ell_{\varepsilon}$ with perfect accuracy. Referring again to Figure 2, what this means is that the pointer quantity $\ell_{\varepsilon}$ by itself provides only an inexact knowledge of the flagpole's actual length. And this brings up another point. Even though the cochannel $\mathbf{M}$ does not specify an unbiased measurement model of $\ell$, in view of $\mathbf{M}\left(\ell_{\varepsilon}\right)=\ell \pm \varepsilon^{\prime}$ with $0 \leq \varepsilon^{\prime} \leq \frac{1}{2}$, it is close enough to being unbiased, so that $\widehat{\ell}$ can still be used as a reliable reconstruction of measurand $\ell$. In this way the cochannel $\mathbf{M}$ - in its role of a measurement model - offers an optimal reconstruction of the measurand, relative to the chosen pointer quantity.

At this stage the reader may feel that our treatment of length measurement has tended to be simple and its technical part ended up being somewhat of an algebraic overkill.
It should be aparent by now that the example we discussed above was meant to illustrate static measurement of a deterministic variety that we believe helps the reader to become aware of a large variety of modeling options. There is a general scheme for length measurement that includes the measurement model we have chosen above. We will pause only to present a variant of such a scheme and will not explore the details further, since they properly belong to Section 4. First, heuristically speaking, Dirac probability measures can alternatively be viewed from the perspective of a measurable mapping of the form $D:[0, L] \longrightarrow \mathbf{P}_{e x}\left([0, L]_{\varepsilon}\right) \subset \mathbf{P}\left([0, L]_{\varepsilon}\right)$ that assigns to each length value $\lambda$ a unique Dirac probability measure $D_{\lambda}$ on $[0, L]_{\varepsilon}$. Secondly, it is then natural to consider more general measurable mappings of the form $T$ : $[0, L] \longrightarrow \mathbf{P}\left([0, L]_{\varepsilon}\right)$, called transition probabilities or Markov kernels, that map each length value $\lambda$ to a unique probability measure $T_{\lambda}$ on $[0, L]_{\varepsilon}$. For example, we may specify $T_{\lambda}$ by stipulating that $T_{\lambda}\left(\ell_{\varepsilon} \bumpeq \Re_{\varepsilon}(\lambda)\right)=p$ with $1>p>0.75$, $T_{\lambda}\left(\ell_{\varepsilon} \bumpeq \Re_{\varepsilon}(\lambda)-\varepsilon\right)=T_{\lambda}\left(\ell_{\varepsilon} \bumpeq \Re_{\varepsilon}(\lambda)+\varepsilon\right)=\frac{1-p}{2}$, and zero otherwise. The basic idea behind the transition probability $T_{\lambda}$ is that, intuitively speaking, the probability of getting the measurement outcome $\Re_{\varepsilon}(\lambda)$ is quite high (above $75 \%$ ) but not certain, since the result could be different from $\Re_{\varepsilon}(\lambda)$ on both sides in the amount of $\pm \varepsilon$ with the remaining probabilities $\frac{1-p}{2}$ and $\frac{1-p}{2}$, respectively, and zero otherwise. Last but not least, in modeling length measurement with error we assume that the target system is in an unknown state encoded by a probability measure $P \in \mathbf{P}([0, L])$ that is mapped to the probabilistic state $\mathbf{M}^{*}(P) \in \mathbf{P}\left([0, L]_{\varepsilon}\right)$, defined by

$$
\left[\mathbf{M}^{*}(P)\right](E)=\int_{[0, L]} T_{\lambda}(E) P(\mathrm{~d} \lambda)
$$

for all events $E$ in $\mathscr{F} \varepsilon$, where $T_{\lambda}$ denotes the discrete probability distribution on possible measurement outcomes, given that the flagpole's actual length is $\lambda$. In general, the transition probability $T_{\lambda}$ models the 'noise' or uncertainty in the readings of measurement. In the dual algebraic setting, the cochannel counterpart

$$
\mathbf{M}: \mathfrak{L}_{\infty}\left([0, L]_{\varepsilon}, \mathscr{F}_{\varepsilon}, P_{\varepsilon}\right) \longrightarrow \mathfrak{L}_{\infty}([0, L], \mathscr{F}, P),
$$

defined by $\mathbf{M}\left(\ell_{\varepsilon}\right)=\widehat{\ell}$ with $\widehat{\ell}(\lambda)=\sum_{l \in[0, L] \varepsilon} \ell_{\varepsilon}(l) \cdot T_{\lambda}(\{l\})$ for all $\lambda$, models the measurand's reconstruction with a statistical error, using the pointer quantity $\ell_{\mathcal{E}}$. Since $T_{\lambda}(\{l\})$ denotes the probability that the flagpole's objective length $\lambda$ is measured by the yardstick in $\varepsilon$ units $a s$ length $l$, in general the measurement error (bias) can be arbitrarily large. However, if the transition probability $T$ is such that $T_{\lambda}(\{l\})=1$ for $\Re_{\varepsilon}(\lambda)=l$ and 0 otherwise, then we obtain the earlier discussed deterministic case.

Another concept that plays an important role in our study of length measurement is that of a comparison of measurement methods. Suppose we have two yardsticks or more generally two different ways of measuring the flagpole's length. The first of these is coarser, using a measurement unit $\varepsilon$ in, say, centimeters, and the second method is finer, with a measurement unit $\varepsilon^{\prime}$ in millimeters, so that we have $\varepsilon^{\prime}<\varepsilon$. The 
commutative diagram below shows the projective (onto) relationships between the parent value space $[0, L]$ and its two descent discretized value spaces.

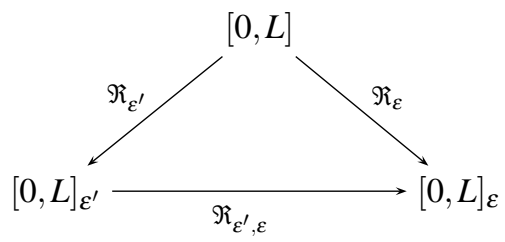

We can readily see that $\Re_{\varepsilon^{\prime}, \varepsilon}\left(\Re_{\mathcal{E}^{\prime}}(\lambda)\right)=\mathfrak{R}_{\varepsilon}(\lambda)$ holds for all $\lambda$. It is of interest to consider the cochannel representations $\mathbf{M}$ and $\mathbf{M}^{\prime}$ of measurements of measurand $\ell$ with the respective pointer quantities $\ell_{\varepsilon}$ and $\ell_{\varepsilon}^{\prime}$ of unequal accuracy, shown in the commutative diagram below:

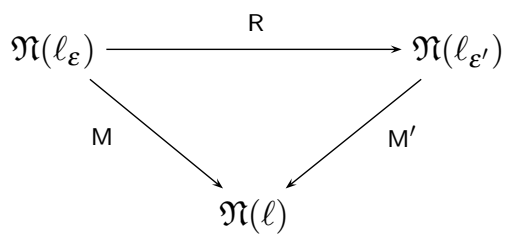

In the diagram above, the connecting map $\mathrm{R}$ handles the refinement relationship between two different methods of measurement. Obviously, the discrete steps of the estimator $\widehat{\ell}^{\prime}$, specified by measurement $\mathbf{M}^{\prime}$, are smaller and therefore closer to $\ell$, than those of $\widehat{\ell}$, determined by the cochannel $\mathbf{M}$.

Having developed a general algebraic approach to static length measurement, we can now ask: What happens to length measurement in the limit, when increasingly more refined yardsticks are used with smaller and smaller measurement units $\varepsilon$, ad infinitum? For example, suppose we set $\varepsilon_{n}=\frac{1}{10^{n}}$ with $n \geq 1$ to specify a sequence of pointer quantities $\ell_{1}, \ell_{2}, \ldots$ with respective round-off accuracies $\frac{1}{10}, \frac{1}{100}, \ldots$, given (say) in decimal fractions of a centimeter. In this way we obtain a corresponding sequence $\mathbf{M}_{1}, \mathbf{M}_{2}, \ldots$ of deterministic measurement models for measuring $\ell$ with increasingly finer pointer quantities $\ell_{n}$, as displayed in the direct limit diagram below. $^{27}$

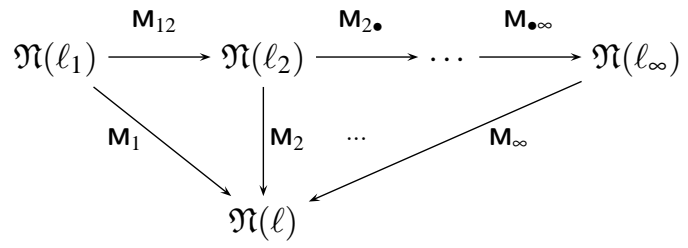

Since von Neumann algebras of random quantities possess excellent convergence properties, the measurement model representing the measurement of $\ell$ with the limit pointer quantity $\ell_{\infty}=\lim _{n} \ell_{n}$ is perfect. Suffice it to say, in summary, that a foundationally important classical criterion for judging the worth of an estimator $\widehat{\ell}$ for measurand $\ell$ is to consider the

\footnotetext{
${ }^{27}$ It must be noted that the analysis presented here is based on the idealizations of classical physics. The so-called Planck length of $1.616 \cdot 10^{-33}$ centimeters renders the quantum mechanical interpretation of the direct limit of the foregoing sequence of discrete quantity algebras meaningless.
}

limit of a nested sequence of estimators as their measurement errors go to zero.

Next, we focus our attention on tensor products of quantity algebras, crucial in the treatment of measurement coupling between target systems and measuring instruments.

\subsection{Tensor products of quantity algebras and compound systems}

Since dynamical measurement of a quantity $f$ - realized in a natural system $S$ we wish to study - is based on a physical interaction between the quantity-bearing system and a measuring instrument $\mathrm{M}(f)$ designed to measure $f$, understanding of the physics of the measurement process under consideration requires a correct mathematical representation of the composite system, henceforth denoted by $\mathrm{S}+\mathrm{M}(f)$. In this subsection we briefly review the pertinent tensor product machinery.

Suppose we have a natural system $S$ and a measuring instrument $\mathrm{M}(f)$, chosen to measure quantity $f$ instantiated by $\mathrm{S}$. Let $\mathfrak{A}_{\mathrm{S}}$ and $\mathfrak{A}_{\mathrm{M}(f)}$ be the respective Banach algebras describing the target system and instrument for $f$. It is well known (see, for example [1]) that the compound system $\mathrm{S}+\mathrm{M}(f)$, including $\mathrm{S}$ and $\mathrm{M}(f)$ as its well-defined subsystems which physically interact with each other for a certain period of time, obeys the fundamental composition law

$$
\mathfrak{A}_{\mathrm{S}+\mathrm{M}(f)} \cong \mathfrak{A}_{\mathrm{S}} \otimes \mathfrak{A}_{\mathrm{M}(f)},
$$

stating that the quantity algebra associated with the 'system + instrument' compound system $\mathrm{S}+\mathrm{M}(f)$ is isomorphic to the (projective) tensor product ${ }^{28}$ of the respective algebras of constituent systems. In the algebra isomorphism above, a quantity $u$ of system $S$ is identified with the quantity $u \otimes 1$ of the compound system $\mathrm{S}+\mathrm{M}(f)$, and likewise a quantity $v$ of $\mathrm{M}(f)$ is identified with $1 \otimes v$. For example, since a measurand $f$ and its pointer quantity $\oslash f$ tend to belong to different algebras, the theoretical error quantity is conveniently defined by their difference $f \otimes 1-1 \otimes \otimes f$ that employs the tensor product. $^{29}$

\footnotetext{
${ }^{28}$ Here there are only two things that we need to know about tensor products of quantity algebras. The first is the tensor calculus of quantities. Simply, if $\left(f_{i}\right)$ and $\left(g_{j}\right)$ are bases of algebras $\mathfrak{A}$ and $\mathfrak{B}$, respectively, then the family $\left(f_{i} \otimes g_{j}\right)$ of simple tensor products is a basis of the tensor product algebra $\mathfrak{A} \otimes \mathfrak{B}$, where $\otimes: \mathfrak{A} \times \mathfrak{B} \longrightarrow \mathfrak{A} \otimes \mathfrak{B}$ is a bilinear map that sends each pair $(f, g)$ in the Cartesian product quantity algebra to the simple tensor quantity $f \otimes g$. Distributivity laws $(f+g) \otimes h=(f \otimes h)+(g \otimes h)$ and $h \otimes(f+g)=(h \otimes f)+(h \otimes g)$ together with the associativity property $c \cdot(f \otimes g)=(c \cdot f) \otimes g=f \otimes(c \cdot g)$ automatically hold for all quantities in the tensor product algebra. In addition, we have $(f \otimes g) \bullet\left(f^{\prime} \otimes g^{\prime}\right)=$ $\left(f \bullet f^{\prime}\right) \otimes\left(g \bullet g^{\prime}\right)$ for all quantities $f, f^{\prime} \in \mathfrak{A}$ and $g, g^{\prime} \in \mathfrak{B}$. The second fact to know about tensor products is their universal property. Concretely, tensor products are specifically designed to turn bilinear maps that do not belong to the category of Banach algebras into legitimate Banach algebra homomorphisms. The empirical justification of tensor products of quantity algebras in algebraic-analytic measurement theory is based on their natural one-to-one correspondence with the Cartesian product of their representing state spaces.

${ }^{29}$ Recall again that symbol 1 denotes the unit of the constituent algebras, encoding the uninformative quantity, having a constant value equal to 1 . In this manner, the algebras of constituent systems can also be seen as independent subalgebras of their tensor product algebra. As may be expected, subal-
} 
From the point of view of physics, instrument-based dynamical measurement of a target system's measurand involves (i) a measurement coupling represented by the tensor product of the target system's quantity algebra and the algebra of the measurand's designated measuring instrument, (ii) joint dynamics thereon, and (iii) interconnection laws between them. It is important to bear in mind that by necessity or by choice, in general not all quantities in $\mathfrak{A}_{\mathrm{S}}$ are suitable for instantaneous measurement, and those that are selected for measurement, are seldom measurable directly. In view of a limited set of feasible measurands and their indirect measurements, real-world measurement procedures are bound to provide only partial information about their target systems. What is of particular measurement-theoretic interest here is an informationally complete subset of measurands. Such subsets completely characterize the measurands' target system at any given instant of time.

Besides tensoring quantity algebras, we must also tensor their (deterministic and probabilistic) state spaces. Suppose we are given expectation functionals $\mathscr{E}$ and $\mathscr{E}_{f}$ (representing states) on algebras $\mathfrak{A}_{\mathrm{S}}$ and $\mathfrak{A}_{\mathrm{M}(f)}$, respectively. Then there is a unique tensor expectation functional $\mathscr{E} \otimes \mathscr{E}_{f}$ on the tensor algebra $\mathfrak{A}_{\mathrm{S}} \otimes \mathfrak{A}_{\mathrm{M}(f)}$, defined by $\left[\mathscr{E} \otimes \mathscr{E}_{f}\right](u \otimes v)=\mathscr{E}(u) \cdot \mathscr{E}_{f}(v)$ for all $u$ in $\mathfrak{A}_{\mathrm{S}}$ and $v$ in $\mathfrak{A}_{\mathrm{M}(f)}$. The probabilistic state represented by the expectation functional $\mathscr{E} \otimes \mathscr{E}_{f}$ is appropriately called the (affine) tensor product of states represented by $\mathscr{E}$ and $\mathscr{E}_{f}$. The (affine) tensor product of states which we have just described can be formulated in the abstract setting of the following commutative diagram:

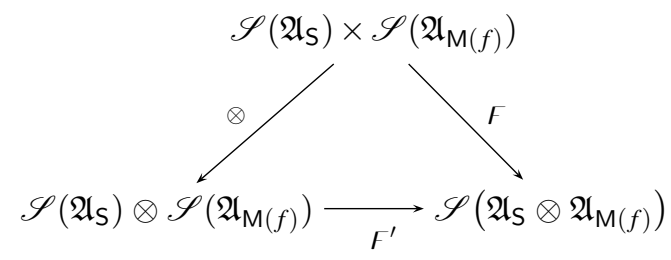

Closely paralleling tensor products of algebras, for every biaffine mapping $\digamma$ (possesses the affine property in both arguments) of states there exists a unique affine mapping $\digamma^{\prime}$ such that $\digamma=\digamma^{\prime} \circ \otimes$.

We said that to dynamically measure the value of a given quantity $f$, it is necessary to allow the target system to interact with a designated measuring instrument of $f$ for a certain period of time. In view of a law-based measurement coupling, the instrument $\mathrm{M}(f)$ behaves in such a way that if the target system is initially in the unknown state $\mathscr{E}_{0}$ and the instrument is in the familiar 'null position' or 'reference' state $\mathscr{E}_{0}^{f}$, then after the interaction is turned on, the composite system $\mathrm{S}+\mathrm{M}(f)$ evolves (ideally after an infinite duration of time) from the initial tensor product state $\mathscr{E}_{0} \otimes \mathscr{E}_{0}^{f}$ into an entangled final tensor product state $\mathscr{E} t$ belonging to $\mathscr{S}\left(\mathfrak{A}_{\mathrm{S}} \otimes \mathfrak{A}_{\mathrm{M}(f)}\right)$, determined by the dynamics of the interaction, terminated at time $t$. Now, this final joint post-measurement state of the

gebras of a quantity algebra can also be used in characterizing subsystems of the target system. Tensor product represents an interactive 'conjunction' of quantities. 'system + instrument' bipartite system determines a unique state $\mathscr{E}_{t} \mid \mathfrak{A}_{\bigcirc f}$ of the measuring instrument $\mathrm{M}(f)$, specified by the following simple restriction operation: $\left[\mathscr{E}_{t} \mid \mathfrak{A}_{\oslash f}\right](v)=d f$ $\mathscr{E}_{t}(1 \otimes v)$ for all instrument quantities $v$. And the 'reduced' instrument state in turn determines the (expected) value of the pointer quantity $\oslash f$, interpreted as the final outcome of measuring quantity $f$. We shall return to this matter in Section 4, where it will be more adequately discussed in the language of information channels.

We round off the present subsection with a brief remark about tensor products of data lattices. Suppose we have a natural system $\mathrm{S}$ with measurand $f$ and a measuring instrument $\mathrm{M}(f)$ chosen for its measurement, characterized by the respective quantity algebras $\mathfrak{A}_{\mathrm{S}}$ and $\mathfrak{A}_{\mathrm{M}(f)}$. We already know that the description of the compound system $\mathrm{S}+\mathrm{M}(f)$ is accomplished by the tensor product quantity algebra $\mathfrak{A}_{\mathrm{S}} \otimes$ $\mathfrak{A}_{\mathrm{M}(f)}$. Here the basic result is the associated data lattice isomorphism law

$$
\mathfrak{L}_{\mathrm{S}+\mathrm{M}(f)} \cong \mathfrak{L}_{\mathrm{S}} \otimes \mathfrak{L}_{\mathrm{M}(f)},
$$

stating that the data lattice associated with the compound system $\mathrm{S}+\mathrm{M}(f)$ is lattice isomorphic to the tensor product of the system's data lattice and the measuring instrument's data lattice. ${ }^{30}$ As in the case of the underlying quantity algebras, the lattices $\mathfrak{L}_{\mathrm{S}}$ and $\mathfrak{L}_{\mathrm{M}(f)}$ are naturally embedded into their tensor product lattice. In particular, a pair of elementary propositions $f \bumpeq c$ (about the system S) and $\oslash f \notin B$ (about the instrument $\mathrm{M}(f)$ ) is mapped to the simple tensor product proposition $(f \bumpeq c) \otimes(\oslash f \Subset B)$ (thought of as a joint proposition) of the bipartite system. We can now analyze the deterministic relationship between a measurand $f$ having the value $c$ at a given time and its pointer quantity $\oslash f$ responding with a determined value in a Borel set $B_{c}$ in terms of implications of the form

$$
(f \bumpeq c) \otimes \top \Longrightarrow \top \otimes\left(\oslash f \notin B_{c}\right)
$$

in the tensor data lattice $\mathfrak{L}_{\mathrm{S}} \otimes \mathfrak{L}_{\mathrm{M}(f)}$, where $T$ denotes the unit element in constituent data lattices. In a law-like manner, this forward relationship links an unknown precise equational item of information about the system's measurand $f$ to a generally less precise item of information about the instrument's pointer quantity $\oslash f$, expressing some form of approximation. To obtain information about the measurand's actual value from the value of its pointer quantity, experimenters must resort to certain inverse methods of estimation. This is not a problem in Bayesian approaches, because in state $\mathscr{E}$ the conditional probability $P_{\mathscr{E}}\left(\oslash f \Subset B_{c} \mid f \bumpeq c\right)$ is intimately linked to its 'causal converse' $P_{\mathscr{E}}\left(f \bumpeq c \mid \oslash f \notin B_{c}\right)$ by the Bayes theorem.

\footnotetext{
${ }^{30}$ The notion of a tensor product of two lattices parellels that of Banach algebras. It is based on a lattice bihomomorphism $\otimes: \mathfrak{L}_{\mathrm{S}} \times \mathfrak{L}_{\mathrm{M}(f)} \longrightarrow$ $\mathfrak{L}_{\mathrm{S}} \otimes \mathfrak{L}_{\mathrm{M}(f)}$ that assigns to pairs $(\Phi, \Psi)$ of propositions in the Cartesian product lattice their tensor product $\Phi \otimes \Psi$ proposition in such a way that the usual distributive laws $(\Phi \vee \Psi) \otimes \Theta=(\Phi \otimes \Theta) \vee(\Psi \otimes \Theta)$ and $\Theta \otimes(\Phi \vee \Psi)=$ $(\Theta \otimes \Phi) \vee(\Theta \otimes \Psi)$ hold for the join operation $\vee$, and likewise for the meet operation $\wedge$.
} 
Since in von Neumann algebra settings of quantity algebras the characteristic functions of the form $1_{\llbracket f \bumpeq c \rrbracket}$ or $1_{\llbracket g \Subset B \rrbracket}$ are two-valued quantities (and so is their tensor product $\left.1_{\llbracket f \bumpeq c \rrbracket} \otimes 1_{\llbracket g \Subset B \rrbracket}=1_{\llbracket f \bumpeq c \rrbracket \times \llbracket g \Subset B \rrbracket}\right)$, data lattices of propositions can be identified with the quantity algebra's lattice of twovalued quantities. The details are omitted since we will not need them.

\subsection{Temporal evolution of quantities in dynamical systems}

In the absence of a time structure, quantity algebras are appropriate for static measurements, such as measuring the diameter of a shaft with a micrometer. However, in the case of quantity algebras of temporally varying quantities and in their continuous measurements (in which information is continually extracted from the system about the measurand), there is a need for an important additional structure, namely, the algebra's temporal dynamics that tells us how quantities change from an earlier time to a later time and how measurement information varies. Mathematically, we are provided with a one-parameter family of maps $\mathfrak{d}_{t}: \mathfrak{A} \longrightarrow \mathfrak{A}$ from the representing Banach algebra $\mathfrak{A}$ to itself, called the target system's time-indexed dynamical transition maps (where the indexing variable $t$ takes its values in a time monoid $\langle\mathbb{T}, 0,+\rangle$, which is usually either the additive monoid of non-negative reals $\left\langle\mathbb{R}_{+}, 0,+\right\rangle$ or the monoid of natural numbers $\langle\mathbb{N}, 0,+\rangle$ ), satisfying the following so-called monoid-action laws for all $t, t^{\prime} \in \mathbb{T}$ and for all quantities $f$ and $g$ in $\mathfrak{A}$ :

(i) Banach algebra endomorphism requirements:
(a) $\mathfrak{d}_{t}(1)=1$,
(b) $\mathfrak{d}_{t}(f+g)=\mathfrak{d}_{t}(f)+\mathfrak{d}_{t}(g)$, and
(c) $\mathfrak{d}_{t}(f \bullet g)=\mathfrak{d}_{t}(f) \bullet \mathfrak{d}_{t}(g) .{ }^{31}$

(ii) Identity: $\mathfrak{d}_{0}=\mathbf{I}_{\mathfrak{A}}$.

(iii) Monoid action: $\mathfrak{d}_{t} \circ \mathfrak{d}_{t^{\prime}}=\mathfrak{d}_{t+t^{\prime}}$

The one-parameter family $\left(\mathfrak{d}_{t}\right)_{t \in \mathbb{T}}$ of dynamical transition maps defined above represents the target system's irreversible (dissipative) deterministic temporal dynamics. In more detail, the transition map $\mathfrak{d}_{t}$ specifies the temporal evolution of quantities by sending a given quantity $f_{0}$ considered at time $t=0$ to the quantity $f_{t}={ }_{d f} \mathfrak{d}_{t}\left(f_{0}\right)$ considered at time $t$ that evolved from $f_{0}$ in a dimensionally homogeneous manner. A Banach algebra $\mathfrak{A}$ equipped with a one-parameter family $\left(\mathfrak{d}_{t}\right)_{t \in \mathbb{T}}$ of dynamical transition maps, which encodes the irreversible action of a time monoid $\mathbb{T}$ on the algebra $\mathfrak{A}$, is called a $\mathbb{T}$-dynamical Banach algebra or simply a dynamical Banach algebra (when the time domain is clear from the context), and in view of the time monoid-action on quantities,

\footnotetext{
31 As is obvious from the linearity requirement, the dynamical evolution of a quantity in essence means a temporal change of its values. As for the temporal evolution of product quantities, they vary in accordance with the temporal changes in their factors. Of course, the unit quantity never changes its value.
}

it is suggestively denoted by $\mathbb{T} \underset{\mathfrak{d}}{\curvearrowright} \mathfrak{A}$. This concept will be used extensively throughout this paper in representing natural time-dependent systems. As an aside, we mention that several additional dynamical algebra structures become available upon passing from time domains to spatial, spacetime or other parameterizing domains, arising, for example, in the context of systems described by partial differential equations.

Transition maps on quantities are often determined by the target system's underlying laws of motion. Classically, the target system's time evolution is given by an ordinary differential equation of the form $\frac{d x}{d t}=\mathrm{F}(\mathrm{x})$ (satisfying the Lipschitz existence condition), in which the unknown quantity is represented by a state-valued function (describing a signal) $\mathrm{x}: \mathbb{T} \longrightarrow \mathscr{X}$ such that its value $\mathrm{x}(t)$ encodes the system's deterministic state at time $t$. We know that the general solution of the equation above involves an arbitrary constant $x_{0}$, determined by the equation's initial condition. Since solutions depend both on the time parameter $t$ and an initial value $x_{0}$, we can represent all of them by a single jointly continuous transition map $\delta: \mathbb{T} \times \mathscr{X} \longrightarrow \mathscr{X}$, satisfying the following continuous monoid-action properties for all time instants $t, t^{\prime} \in \mathbb{T}$ and for all states $\mathrm{x}$ in $\mathscr{X}$ :

(i) Identity property: $\delta(0, \mathrm{x})=\mathrm{x}$, and

(ii) Group property: $\delta\left(t, \delta\left(t^{\prime}, \mathrm{x}\right)\right)=\delta\left(t+t^{\prime}, \mathrm{x}\right)$.

As in the case of dynamical Banach algebras, a monoid time-domain $\mathbb{T}$ acting on a topological state space $\mathscr{X}$, specified by a jointly continuous transition map $\delta$ satisfying the monoid-action properties listed above, defines a (deterministic) topological dynamical model, denoted $\mathbb{T} \underset{\delta}{\curvearrowright} \mathscr{X}$. Our interest in topological dynamical models is motivated by their Gelfand-style relationship to dynamical Banach algebras. Specifically, the foregoing jointly continuous transition map $\delta$ on the state space $\mathscr{X}$ induces a unique family of dynamical transition maps of the form $\mathfrak{d}_{t}: \mathbf{C}(\mathscr{X}) \longrightarrow \mathbf{C}(\mathscr{X})$ on the Banach algebra $\mathbf{C}(\mathscr{X})$ of continuous real-valued functions on $\mathscr{X}$, defined by $\mathfrak{d}_{t}\left(g_{0}\right)={ }_{d f} g_{t}$, where $g_{t}(\mathrm{x})=g_{0}(\delta(t, \mathrm{x}))$ for all $x$.

There is also an induced (dual) dynamics on states, having the form of affine (convex) endomorphisms $\mathfrak{d}_{t}^{*}: \mathscr{S}(\mathbf{C}(\mathscr{X})) \longrightarrow \mathscr{S}(\mathbf{C}(\mathscr{X}))$ on probabilistic states (preserving their convex combinations), defined by the composition $\mathfrak{d}_{t}^{*}(\mathscr{E})=\mathscr{E} \circ \mathfrak{d}_{t}$ for all states $\mathscr{E}$. Under this dynamics, a state $\mathscr{E}_{t}$ at time $t$ evolves into the state $\mathscr{E}_{t+t^{\prime}}=\mathfrak{d}_{t^{\prime}}^{*}\left(\mathscr{E}_{t}\right)$ for all $t^{\prime} \geq 0$. Importantly, this dynamics induces in turn a dynamics on the space $\mathscr{S}_{e x}(\mathfrak{A})$ of extremal expectation functionals (representing deterministic states), so that by the Riesz representation theorem we are permitted to return to the topological dynamics $\delta(t, \cdot): \mathscr{X} \longrightarrow \mathscr{X}$ we have started from. This implies that we may work, as convenient, in a geometric setting with topological dynamical models of the form $\mathbb{T} \underset{\delta}{\curvearrowright} \mathscr{X}$ or in an algebraic framework with corresponding dynamical Banach algebras of the form $\mathbb{T} \curvearrowright \mathbf{C}(\mathscr{X})$. The fact that a modeler deals with the dynamics on the probabilistic 
state space $\mathscr{S}(\mathbf{C}(\mathscr{X}))$ rather than on the deterministic state space $\mathscr{X}$ is directly related to his or her ignorance about the target system's precise initial state.

Of central importance is the construction of tensor products of dynamical Banach algebras. As we shall see, the tensor product

$$
\left(\mathbb{T} \underset{\mathfrak{d}}{\curvearrowright} \mathfrak{A}_{\mathrm{S}}\right) \otimes\left(\mathbb{T} \underset{\mathfrak{d}^{\prime}}{\curvearrowright} \mathfrak{A}_{\mathrm{S}^{\prime}}\right)=\mathbb{T} \underset{\mathfrak{d} ; \mathfrak{d}^{\prime}}{\curvearrowright} \mathfrak{A}_{\mathrm{S}} \otimes \mathfrak{A}_{\mathrm{S}^{\prime}}
$$

of dynamical Banach algebras $\mathbb{T} \underset{\mathfrak{d}}{\curvearrowright} \mathfrak{A}_{\mathrm{S}}$ and $\mathbb{T} \underset{\mathfrak{d}^{\prime}}{\underset{A^{\prime}}{\curvearrowright}} \mathfrak{A}_{\mathbf{S}^{\prime}}$ is the cornerstone of the algebraic model of measurement coupling between a time-varying target system $S$ and a measuring instrument $S^{\prime}$. As expected, the transition map $\mathfrak{d} ; \mathfrak{d}^{\prime}$ on the tensor product $\mathfrak{A}_{\mathrm{S}} \otimes \mathfrak{A}_{\mathcal{S}^{\prime}}$ algebra is given by the tensor product $\mathfrak{d} \otimes \mathfrak{d}^{\prime}$ of constituent transitions. Later on, we shall be discussing several applications of tensor product quantity algebras. At this stage, however, we briefly mention just one, touched upon earlier.

Algebraic approaches to measurement error usually rely on a comparison of the measurand's $f$ actual values with the measured values of its associated pointer quantity $\oslash f$. However, since $f$ is an element of the target system's quantity algebra $\mathfrak{A}_{S}$ and its pointer quantity $(7 f$ belongs to the instrument's algebra $\mathfrak{A}_{\mathrm{M}(f)}$ designed to measure $f$, no straightforward comparison makes mathematical sense. Nevertheless upon passing to the measurement dynamics

$$
\mathfrak{A}_{\mathrm{S}} \otimes \mathfrak{A}_{\oslash f} \stackrel{\mathfrak{m}_{t}}{\longrightarrow} \mathfrak{A}_{\mathrm{S}} \otimes \mathfrak{A}_{\oslash f}
$$

on the tensor product algebra $\mathfrak{A}_{\mathrm{S}} \otimes \mathfrak{A}_{\mathrm{M}(f)}$, the much needed comparison is readily expressed by the tensorial measurement error quantity

$$
1 \otimes \oslash f_{t}-f_{0} \otimes 1
$$

in $\mathfrak{A}_{\mathrm{S}} \otimes \mathfrak{A}_{\mathrm{M}(f)}$, given by the difference between the temporally evolved pointer quantity $\oslash f_{t}$ under dynamics $\mathfrak{m}_{t}$ at time instant $t>0$ immediately following the termination of measurement and the measurand $f_{0}$ at the pre-measurement time instant $t=0$, immediately preceding the act of measurement. Unfortunately, because the actual value of the measurand $f_{0}$ cannot be known exactly, it is usually replaced with an operationalized value, that can (at least in principle) be obtained by the most accurate measurement method.

Several important classical measurement processes can now be put into the above dynamical Banach or von Neumann algebra framework. As a simple application of tensor products, we now take up the example of length measurement, discussed in some detail in Subsection 2.3. Recall that in modeling the measurement a flagpole's length, using a yardstick as our designated measuring tool, we relied on von Neumann algebras $\mathfrak{N}(\ell)$ and $\mathfrak{N}\left(\ell_{\varepsilon}\right)$, finitely generated by the length quantity $\ell$ and its associated pointer quantity $\ell_{\varepsilon}$, respectively. To obtain a workable model of the pertinent measurement procedure, it became necessary to pass to the corresponding state space frameworks $\mathscr{S}(\mathfrak{N}(\ell))$ and $\mathscr{S}\left(\mathfrak{N}\left(\ell_{\varepsilon}\right)\right)$.

To understand the flagpole-yardstick measurement interaction better and in order to bring our reasoning about a large variety of measurement processes under a common conceptual umbrella, we now consider an automorphism $\mathfrak{m}^{*}$ : $\mathscr{S}\left(\mathfrak{N}(\ell) \otimes \mathfrak{N}\left(\ell_{\varepsilon}\right)\right) \longrightarrow \mathscr{S}\left(\mathfrak{N}(\ell) \otimes \mathfrak{N}\left(\ell_{\varepsilon}\right)\right)$ that models the passage from a pre-measurement state $D_{\lambda} \otimes D_{0}$ (when the flagpole and yardstick were not yet engaged in any act of measurement) to a post-measurement state $D_{\lambda} \otimes D_{\mathfrak{R}_{\varepsilon}(\lambda)}$ (when the measurement is complete and ready for readout). Obviously, we set $\mathfrak{m}^{*}\left(D_{\lambda} \otimes D_{0}\right)=_{d f} D_{\lambda} \otimes D_{\mathfrak{R}_{\varepsilon}(\lambda)}$ for all length values $\lambda$ in $[0, L] .{ }^{32}$

A fundamental algebraic feature of interactive instrumentbased measurements is captured by the commutative diagram

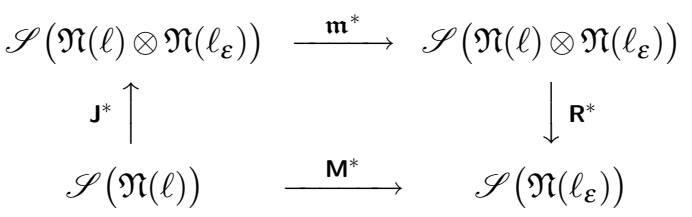

in which the flagpole's initial (pre-measurement, deterministic) state $D_{\lambda} \in \mathscr{S}(\mathfrak{N}(\ell))$ encodes its unknown objective length $\lambda$, mapped by $\mathbf{J}$ to the tensor state $D_{\lambda} \otimes D_{0}$ that includes the yardstick's 'null' or 'reference' state $D_{0}$. As we have already remarked, in mirroring the length measurement procedure, the endomorphism $\mathfrak{m}^{*}$ maps the unknown initial state to the final joint state $D_{\lambda} \otimes D_{\mathfrak{R}_{\varepsilon}(\lambda)}$ that retains the flagpole's length unchanged. However, it appropriately modifies the yardstick's readout state. An important final step is the extraction of the measuring instrument's known state from the joint state. As explained above, this is accomplished by the restriction map $\mathbf{R}^{*}$ (emulating the assignment of marginal probability measures), defined by $\mathbf{R}^{*}\left(D_{\lambda} \otimes D_{\mathfrak{R}_{\varepsilon}(\lambda)}\right)=D_{\mathfrak{R}_{\varepsilon}(\lambda)}$. It is elementary to check that the measurement model (formally a quantity channel) $\mathbf{M}^{*}$, introduced in Subsection 2.3 , is now specified by the composition $\mathbf{M}^{*}=\mathbf{R}^{*} \circ \mathfrak{m}^{*} \circ \mathbf{J}^{*}$, obtained from the diagram above. As we shall see, upon appropriately changing the dynamics, the foregoing diagram automatically carries over to other vastly more involved interactive instrument-based measurement processes. But first, before we embark on a channel-theoretic approach to measurement, we outline a few concepts from discretization theory, which are needed for the presentation of various measurement models.

\section{CONTINUUM AND DISCRETE MODELS OF MEASURING SYSTEMS}

In pursuits of powerful technical results under well-chosen tractability and complexity constraints, most advanced mathematical models of natural dynamical systems rely on the assumption that their underlying domains of time, space and states are furnished with the structure of a topological continuum, locally homeomorphic to $\mathbb{R}^{n}$ for some $n \geq 1$ or to

\footnotetext{
${ }^{32}$ Since this is a trivial temporal evolution, from an off moment of measurement to the completed on moment, the time monoid $\mathbb{T}$ is given by the two element $\{0,1\}=\mathbb{Z}_{/ 2}$ cyclic group, so that $\mathfrak{m}_{1}^{*}=\mathfrak{m}^{*}$ and $\mathfrak{m}_{0}^{*}=$ identity. Under this representation, static measurement can be viewed as a degenerate case of interactive dynamical measurement. Because probabilistic states are convex superpositions of deterministic states represented by Dirac probability measures, extension to more general states is essentially routine.
} 
its compactified variants under the natural topology. In the literature on mathematical modeling it is rarely noticed that this ontologically driven structural assumption about mathematical models of dynamical systems is at odds with a crucial epistemic finiteness condition, capturing the finiteness of all physical resources (manifested in available time, memory, laboratory size, measurement, etc.), necessary for the acquisition, transmission and storage of information pertaining to the target system's quantities. It becomes immediately obvious that any geometric point chosen at random from any of the continuum domains of mathematical models will have, with Lebesgue probability measure equal to one, at least one (nonalgebraic) transcendental coordinate that no normal measurement process can ever identify with absolute accuracy. Models framed in this way raise challenging questions about the empirical relevance of ideal geometric points. Clearly, a measuring instrument designed to measure a quantity $f$ with a continuum value space $\operatorname{Val}(f)$ will need a continuum state space for a perfect measurement of each value of $f$. However, since such an instrument can only display a finite measurement outcome in finite time, the result will at best be a small rational number. A major conceptual issue in the theory of mathematical modeling of natural dynamical systems is bridging the gap between ontologically motivated continuum models and epistemically necessitated discrete models encountered in computation and measurement.

In this section we introduce the notion of discretization of continuum dynamical models and study the relationships between a given so-called parent continuum model and its descent (offspring) discrete models, constructed by suitable discretization methods in the ambience of measurement processes and prediction. We start by considering a continuum model representing a natural dynamical system. This setting is general enough to accommodate the characterization of measured and measuring systems. Next, we show how to discretize the model's underlying time domain, state space, and dynamics thereon. Of course, these ingredients can be discretized in different ways, using different time steps and spatial mesh sizes. The resulting descent discrete models will in general be quite different and will approximate the parent continuum model in different ways. The opposition between continuum and discrete models is not a challenging problem as long as they agree asymptotically or in the limit in the sense that a given sequence of descent discrete models rapidly approaches its parent continuum model as the discretization parameter goes to zero, so that the former can be used as a more or less crude approximation of the latter. Unfortunately, as we shall see, there are many kinds of limits to consider and most of them fail to bring the epistemic and ontological perspectives together. Discretizing smooth and continuum models is considerably more subtle than what one may initially think. As we have already indicated, continuum dynamical models provide a perfect ambience in which the concept of Gelfand duality between topological dynamical models and dynamical Banach algebras operates successfully, and this is also true in the framework of their discretization. For example, discretiza- tion of state spaces turns out to be dual to the discretization of Banach algebras of quantities. Since von Neumann algebras abstract away all continuity and smoothness properties, they serve the objectives of discretization particularly well.

Returning to the general situation, given a target system's measurand $f$ with a continuum value space $\operatorname{Val}(f)$, the reading scale of an ideal instrument for pointer quantity $\oslash f$ is also a continuum, captured by $\operatorname{Val}(\oslash f)$. However, the epistemic finiteness condition tells us that a normal measuring instrument's pointer module can only have a finite number of detectable states and that the measurement outcome associated with a pointer module state is bound to be a rational number. Thus, in an attempt to read and record the value of $\oslash f$, the experimenter has no choice but divide its value space into tiny intervals and then decide which interval actually contains the measured value. In this way, the reading scale of a normal instrument determines only a discrete, coarse-grained version of $\oslash f$, denoted by $\oslash_{\varepsilon} f$ and called a discretization of pointer quantity $\oslash f$ with discretization parameter (level of resolution) $\varepsilon$. Simply, the mathematical continuum of an ideal measuring instrument dial is observationally accessible only in its discretized form.

Thus, we can conclude that researchers must distinguish between ontologically driven mathematical representations of target systems in the form of parent continuum models on one side, and prediction- and measurement-based tools in the form of descent discrete models on the other side. These distinctions are intimately related to the notion of model validation. This brings us to a brief review of basic discretization methods of time domains, state spaces and quantity algebras. We begin by describing the passage from continuum-time dynamical models to discrete-time dynamical models.

\subsection{Temporal discretization of continuum dynamical mod- els}

From the standpoint of physical measurement, most (if not all) time instants are inherently beyond precise observation. Likewise, from the standpoint of computer analysis, the ideal elements of classical spaces (based on a continuum) are accessible only partially in terms of finitary approximations. Therefore, for purposes of measurement and numerical analysis, the continuum-time domain $\langle\mathbb{T}, 0,+\rangle$, henceforth assumed to be homeomorphic to the monoid $\left\langle\mathbb{R}_{+}, 0,+\right\rangle$ of nonnegative reals, has to be replaced by a family of informationtheoretically tractable discrete-time monoids $\langle\tau \mathbb{N}, 0,+\rangle$ with a suitable time-step (sampling) parameter $\tau>0$, where $\tau \mathbb{N}=$ $\{0, \tau, 2 \tau, \cdots\}$ is a submonoid of $\mathbb{R}_{+}$, comprised of discrete time-steps (i.e., integer multiples of tau, measured in milliseconds, minutes, days, etc.), determined by experimental sampling regimes.

Clearly, to extract a workable finitary information from a parent continuum-time dynamical model $\mathbb{R}_{+} \curvearrowright \mathscr{X}$ of a target system about its phase portrait, some form of time domain discretization is necessary. ${ }^{33}$

\footnotetext{
${ }^{33}$ Recall that even in classical systems science, solutions of (nonlinear) dif-
} 
Quite simply, the $\tau$-time discretization of a parent continuum-time dynamical model $\mathbb{R}_{+} \underset{\delta}{\curvearrowright} \mathscr{X}$ is defined by the time domain restriction

$$
\left(\mathbb{R}_{+} \underset{\delta}{ } \mathscr{X}\right)_{\left.\right|_{\mathbb{N}}}={ }_{d f} \tau \mathbb{N} \underset{\delta_{\tau}}{\curvearrowright} \mathscr{X},
$$

of $\mathbb{R}_{+}$to $\tau \mathbb{N}$, where $\delta_{\tau}(\tau k, \mathrm{x})={ }_{d f} \delta(\tau k, \mathrm{x})$ for all natural numbers $k$ and states $\mathrm{x}$ in $\mathscr{X}$. Clearly, when a continuous time function is sampled in discrete time-steps $\tau, 2 \tau, 3 \tau, \cdots$, in general, there will be a loss of information that depends on the size of the time-step. In applications, it is important to identify sufficiently good discrete-time approximations of the parent continuum-time model's trajectories and other dynamical objects. As a matter of further interest, it is easily verified that there is a transformation on models that transforms each parent continuum-time dynamical model $\mathbb{R}_{+} \underset{\delta}{\curvearrowright} \mathscr{X}$ into its discrete-time variant $\tau \mathbb{N} \underset{\delta_{\tau}}{\curvearrowright} \mathscr{X}$ with a positive time-step parameter $\tau$. Importantly, note that the overall intended interpretation now spreads over two distinct universes of models, namely, the world of parent continuum-time dynamical models and that of descent discrete-time dynamical models.

For example, in the familiar continuum dynamical model of a simple pendulum, the temporal (and spatial) discretization of smooth trajectories, having the geometric form of ellipses, results in discrete ellipses, as shown in Figure 3 below, where the discreteness is controlled by the size of the time-step $\tau$ :

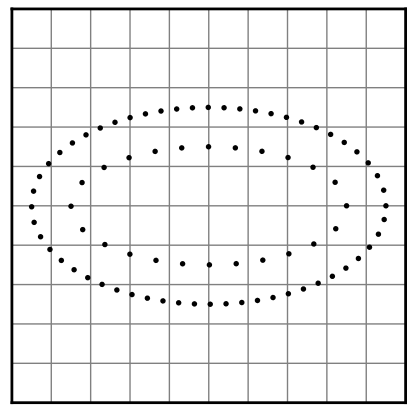

Fig. 3 Temporally discretized elliptical trajectories using two different time steps in a 2-dimensional Cartesian state space

A possible close fit between a parent continuum-time dynamical model and its associated sequence of (descent) temporally discretized dynamical models is established by an isomorphism between the parent model and the inverse limit of the chosen sequence of descent discrete-time dynamical models, as the positive time-step $\tau$ parameter approaches zero. It is important to bear in mind that a parent continuum-time dynamical model $\mathbb{R}_{+} \curvearrowright \mathscr{X}$ cannot be directly linked to any of its descent discrete-time dynamical models of the form $\tau \mathbb{N} \curvearrowright \mathscr{X}$, because these models belong to entirely different categories.

ferential equations often prompt approximations by discrete-time difference equations. Furthermore, the solutions of analytically given differential equations are usually meant to refer to the underlying 'reality' of target system behaviors, and the associated discretized variants are introduced to capture that 'reality' in an (approximate) epistemically accessible manner.
Nevertheless, their empirical interpretations usually overlap or are closely related.

Since time discretization does not seem to be a serious technical problem, in what follows, we shall tacitly assume that it has already been completed and we shall focus exclusively on discrete-time (smooth, topological, etc.) deterministic dynamical models.

\subsection{Spatial discretization of continuum dynamical models}

When a system of differential equations is nonlinear, it will in general be impossible to solve its equations analytically. Computer solutions inevitably bring in the effects of finiteness, round-off, and other truncation operations that can sometimes be very drastic, making the comparisons of computed and 'true' solutions questionable. In a top-down analytic setting, typical in engineering modeling, model construction in the form of equations proceeds in two interleaved phases. First, a system of (differential, difference, etc.) equations is derived or determined in some analytic form. Then, second, its parameter (coefficient) values are estimated from available measurement data. If the equations are incorrect, no coefficient values can make the solutions' predictions match the target system's measurement data. Simply, the equations will have to be revised (e.g., by passing from linear to quadratic equations). In general, these activities are not performed in the world of continuum models. However, upon passing to the solution spaces of differential (difference) equations, we obtain a rigorous universal basis for state space discretization.

Given a temporally discretized variant $\tau \mathbb{N} \underset{\delta_{\tau}}{\curvearrowright} \mathscr{X}$ of a topological dynamical model $\mathbb{R}_{+} \underset{\delta}{ } \mathscr{X}$, where $\mathscr{X}$ is a compact subset of $\mathbb{R}^{n}$ (for some $n \geq 1$ ), in the simplest situation the associated (descent) discrete state space $\mathscr{X}_{\varepsilon}$ is defined by an $n$-dimensional countable lattice (grid) $\mathscr{X}_{\varepsilon}=(\varepsilon \mathbb{Z})^{n} \cap \mathscr{X}$ of homogeneously spaced vectors with a positive space-step $\varepsilon$ in all $n$ directions, serving as centers of an $n$-dimensional mesh of cells (squares, cubes, hypercubes or $n$-cubes in general) of uniform size $\varepsilon .^{34}$ In this way, finitary information about the continuum model's topological state space $\mathscr{X}$ (and associated phase portrait) is extracted by identifying the pertinent cells in which the ideal elements of $\mathscr{X}$ are presumed to lie. Since the cell size and the number of cells have a direct bearing on computational costs, investigators prefer to choose a relatively small continuum dynamical submodel $\mathbb{R}_{+} \curvearrowright_{\delta} \mathscr{X}^{\prime}$ of the continuum parent model $\mathbb{R}_{+} \underset{\delta}{\curvearrowright} \mathscr{X}$ on a (compact) subspace $\mathscr{X}^{\prime}$ of $\mathscr{X}$ - dictated by importance and interest - and proceed to study its localized phase portrait. In this case the submodel's descent discrete space is finite. To identify the overall dynamics and soundness of the parent model, several special submodels of $\mathbb{R}_{+} \curvearrowright \mathscr{X}$ are investigated.

There are many ways to construct a cell structure over the

\footnotetext{
${ }^{34}$ Here and below we adopt the usual notation $\varepsilon \mathbb{Z}=$ $\{\ldots,-2 \varepsilon,-\varepsilon, 0, \varepsilon, 2 \varepsilon, \ldots\}$.
} 
underlying state space of a continuum dynamical model. ${ }^{35}$ For example, suppose the parent continuum dynamical model represents the behavior of a simple pendulum and the investigator's main interest is in the verification of one of its elliptical trajectories (forming a period-one cycle), specified by some initial state and a parameter value. For this purpose, it suffices to confine the parent continuum model's state space to a smaller two-dimensional rectangular (compact) subspace $\mathscr{X} \subset \mathbb{R}^{2}$ of position-velocity points, say, $\mathscr{X}=$ $[-L, L] \times\left[-L^{\prime}, L^{\prime}\right]$. The subspace $\mathscr{X}$ together with its homogeneous grid of size $\varepsilon=0.25$ inches is illustrated in Figure 4 below.

In approximating the elliptical trajectory by a discretization algorithm, the investigator is confronted with the problem of deciding on a mesh, assessing the quality of approximation with that mesh, and then adjusting or refining it as needed. Each chosen positive space-step $\varepsilon$ determines a unique $\varepsilon$-cell

$$
\ominus_{\varepsilon}={ }_{d f}\left\{\left(x_{1}, x_{2}\right) \mid-\frac{\varepsilon}{2} \leq x_{1}<\frac{\varepsilon}{2} \& \frac{\varepsilon}{2} \leq x_{2}<\frac{\varepsilon}{2}\right\},
$$

which is geometrically a half-open square of size $\varepsilon \times \varepsilon$, centered in the origin of the subspace $\mathscr{X} \subset \mathbb{R}^{2}$. This cell can be moved anywhere in the state space $\mathscr{X}$ by translation. For instance, the cell defined by $\odot_{\varepsilon,(p, v)}={ }_{d f}(\varepsilon p, \varepsilon v)+\ominus_{\varepsilon}$, which is easily seen to be equal to the square

$$
\begin{array}{r}
\left\{\left(x_{1}, x_{2}\right) \mid\left(p-\frac{1}{2}\right) \varepsilon \leq x_{1}<\left(p+\frac{1}{2}\right) \varepsilon\right. \\
\left.\&\left(v-\frac{1}{2}\right) \varepsilon \leq x_{2}<\left(v+\frac{1}{2}\right) \varepsilon\right\}
\end{array}
$$

is centered in the lattice point $(\varepsilon p, \varepsilon v)$ of $\varepsilon \mathbb{Z} \times \varepsilon \mathbb{Z}$. For concreteness, let $\mathscr{X}_{\varepsilon}$ be the set of lattice points of the form $(\varepsilon p, \varepsilon v)$ in $(\varepsilon \mathbb{Z} \times \varepsilon \mathbb{Z}) \cap\left([-L, L] \times\left[-L^{\prime}, L^{\prime}\right]\right)$.

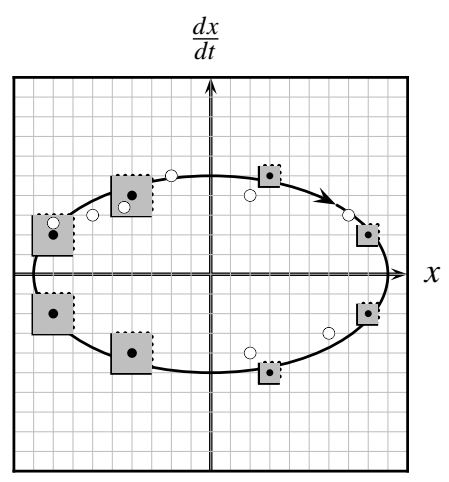

Fig. 4 Refinement of state space discretizations

In Figure 4, cells are displayed using two different mesh sizes: larger (mesh size $\varepsilon$ ) on the left and more refined (mesh

\footnotetext{
${ }^{35}$ In the case of abstract topological state spaces $\mathscr{X}$ the starting point is a coarse graining in the form of a (measurable, open, etc.) partition (covering, tessellation) that induces an equivalence relation on $\mathscr{X}$. Then the representative points (nerves) of the corresponding abstraction classes determine a discrete set of points, giving a locally 'averaged' information about the points in $\mathscr{X}$. In this general setting, a major problem is that these types of equivalence relations fail to be dynamical congruence relations.
}

size $\varepsilon^{\prime}<\varepsilon$ ) on the right. The empty circles illustrate measurement results from validation experiments, important in evaluating the discrepancy between predicted and measured quantity values, specifying states.

Next, we need an $\varepsilon$-discretization map $\mathfrak{R}_{\varepsilon}: \mathscr{X} \longrightarrow \mathscr{X}_{\varepsilon}$ that sends each state $\mathrm{x}$ in the continuum state space $\mathscr{X}$ to its nearest discrete state $\mathfrak{R}_{\varepsilon}(\mathrm{x})$ in the associated discretized state space $\mathscr{X}_{\varepsilon}$. In general, the structural ingredients of a descent spatially discretized model of the parent continuum dynamical model $\tau \mathbb{N} \curvearrowright \mathscr{X}$ of interest are defined as follows:

(i) Discretized state space: For a given (usually small) space-step (grid size, mesh or lattice spacing) $\varepsilon>0$ we define the $\varepsilon$-grid or $\varepsilon$-discretization ( $n$-dimensional $\varepsilon$ lattice) of the parent state space $\mathscr{X} \subset \mathbb{R}^{n}$ as the set

$$
\mathscr{X}_{\varepsilon}={ }_{d f} \mathscr{X} \cap(\varepsilon \mathbb{Z})^{n} .
$$

As signalled above, the descent spatially discretized state space $\mathscr{X}_{\varepsilon}$ comes with its projective discretization map $\mathfrak{R}_{\varepsilon}: \mathscr{X} \longrightarrow \mathscr{X}_{\varepsilon}$, defined by $\mathfrak{R}_{\varepsilon}(\mathrm{x})={ }_{d f}(\varepsilon p, \varepsilon v)$ with $\mathrm{x} \in$ $\bullet_{\varepsilon,(p, v)}$ for all $\mathrm{x}$ in $\mathscr{X}$. We have already indicated that the discretization (round-off) map sends each state $\mathrm{x}$ in the parent continuum model's state space $\mathscr{X}$ to a unique point in its $\varepsilon$-integer lattice subspace $\mathscr{X}_{\varepsilon}$ that is closest to it. Naturally enough (in accordance with a computer discretization procedure), each point is 'rounded off' by its approximant in the integer lattice. This is how the investigator obtains information about the parent model's states or more generally about the phase portrait's trajectories and other dynamical objects. In the reverse direction we have the dual injective dediscretization map $\mathfrak{I}_{\varepsilon}: \mathscr{X}_{\varepsilon} \longrightarrow \mathscr{X}$ satisfying $\mathfrak{R}_{\varepsilon} \circ \mathfrak{I}_{\varepsilon}=\mathbf{I}_{\mathscr{X}_{\varepsilon}}$.

(ii) Discretized dynamics: Given a continuum-space dynamics $\delta: \tau \mathbb{N} \times \mathscr{X} \longrightarrow \mathscr{X}$, its discretized transition map $\delta_{\varepsilon}: \tau \mathbb{N} \times \mathscr{X}_{\varepsilon} \longrightarrow X_{\varepsilon}$ is defined by the base diagram

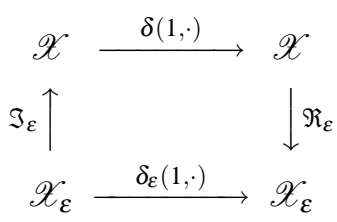

and then extended to all discrete time steps by iterations $\delta_{\varepsilon}(n+1, \mathrm{x})=\delta_{\varepsilon}\left(n, \delta_{\varepsilon}(1, \mathrm{x})\right)$ for all $n$ and $\mathrm{x}$.

It is easy to verify that in the case of the pendulum example discussed above we have

$$
\delta_{\varepsilon}(1,(\varepsilon p, \varepsilon v))=\Re_{\varepsilon}(\delta(1,(\varepsilon p, \varepsilon v))),
$$

specifying a map $\delta_{\varepsilon}(1, \cdot): \mathscr{X}_{\varepsilon} \longrightarrow \mathscr{X}_{\varepsilon}$ that is quickly extended to all discrete time instants by iteration.

Given a descent spatially and temporally discretized dynamical model $\tau \mathbb{N} \curvearrowright_{\delta_{\tau, \varepsilon}} \mathscr{X}_{\varepsilon}$ of a continuum dynamical model $\tau \mathbb{N} \curvearrowright$ $\mathscr{X}$, stroboscopic measurement results of the pendulum's positions and velocities at discretized times can be entered into 
the cell diagram, as shown in Figure 4, and make comparisons with discretized trajectories, given by the parent continuum dynamical model.

As in the case of temporal discretization, the success of spatial discretization depends on the behavior of (inverse) limits of converging sequences of descent spatially discretized dynamical models, as $\varepsilon$ goes to zero. Comparing spatial discretization with temporal discretization, we see that a major technical problem in spatio-temporal discretization is the correct choice of a double limit, as the time step and mesh size approach zero. Here the essential idea is to let the sequence of spatial discretizations to be at least logarithmically far ahead of the sequence of temporal discretizations, so as to allow the dynamics of the latter to correctly converge to the continuum parent model's dynamics. (See [2] for a more detailed discussion of why the succession of temporal vs. spatial discretizations can not be interchanged.)

Because the parent continuum dynamical model's states are not observable directly, they must be identified indirectly in terms of (smooth, continuous, measurable, etc.) quantities, whose values are obtained via measurement. Thus, we also need to look at approximating rational-valued functions of the form $f_{\varepsilon}: \mathscr{X}_{\varepsilon} \longrightarrow \mathbb{Q}$ that are extendable by extrapolation to functions $\bar{f}_{\varepsilon}: \mathscr{X} \longrightarrow \mathbb{R}$ on the entire state space $\mathscr{X}$. The details are investigated further in the next subsection, where we consider Genfand representation in the context of discretization theory.

Every elliptical trajectory in the pendulum dynamical model is reconstructed from a discrete trajectory of a descent discrete model via subsequent refinement, as illustrated in Figure 5.

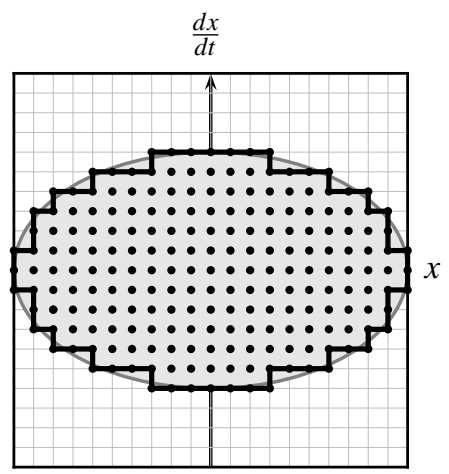

Fig. 5 Discretizing a smooth pendulum trajectory

In this manner, we obtain a descent spatially discretized dynamical model $\tau \mathbb{N} \underset{\delta_{\tau, \varepsilon}}{\curvearrowright} \mathscr{X}_{\varepsilon}$ (with space-step $\varepsilon$ ) of the parent dynamical model $\tau \mathbb{N} \curvearrowright \mathscr{X}$. As alluded to earlier, dynamicists are interested in the asymptotic behavior of the discretized dynamical model $\tau \mathbb{N} \curvearrowright_{\delta_{\tau, \varepsilon}} \mathscr{X}_{\varepsilon}$, as the mesh size $\varepsilon$ goes to zero. It is well known that discretized models even with tiny mesh sizes $\varepsilon$ are not very helpful deterministically, since the correlation between the behavior of the discretized models $\tau \mathbb{N} \underset{\delta_{\tau, \varepsilon}}{\curvearrowright} \mathscr{X}_{\varepsilon}$ (with varying $\varepsilon$ ) and that of the parent continuum dynamical model $\tau \mathbb{N} \underset{\delta}{ } \mathscr{X}$ is usually very weak. Concretely, the descent discretized dynamical model will generate a variety of discretization-dependent spurious and 'phantom' dynamical regimes (periodic orbits, fixed points, etc.) and other artifacts that do not correspond to any behavior present in the parent model. The investigators need to recognize that descent discrete dynamical models are radically different models that may approximate their parent model in some circumstances, but nevertheless they tend to possess properties (e.g., degrees of freedom) and structure that are patently inconsistent with those instantiated by the parent model. The difficulty has to do with the fact that discretization algorithms usually break the parent continuum dynamical model's infinitesimal symmetries, invariant under smooth or continuous dynamical isomorphisms. This problem would not matter too much, if one had a well-defined way of taking the limit of a descent sequence of discretized dynamical models - in which state space and time discretizations go to zero - that would ensure not only a gradual disappearance of inconsistencies (manifested by spurious regimes) but also a total reconstruction of the parent continuum model, modulo dynamical isomorphism. Unfortunately, presently there are no known general necessary and sufficient conditions for the existence of a limit of a descent sequence of discretized dynamical models that faithfully reproduces the parent dynamical model.

\subsection{Algebraic discretization}

Throughout this work we have emphasized the fact that experimenters do not have direct access to the states of their target dynamical systems. To obtain a workable model, it is necessary to characterize the states indirectly, in terms of certain observable quantities, so that instead of observing a state encoded by a point $\mathrm{x} \in \mathscr{X}$ of a representing dynamical model $\tau \mathbb{N} \curvearrowright \mathscr{X}$, experimenters measure the values of smooth, continuous, or measurable observables of the form $f: \mathscr{X} \longrightarrow \mathbb{R}$ in state $x$, sufficient to generate informative data propositions about $x$.

As a prelude to algebraic discretization, recall that in a topological setting each (discrete-time) continuum dynamical model $\tau \mathbb{N} \underset{\delta}{\curvearrowright} \mathscr{X}$ comes with its associated dynamical Banach algebra model $\tau \mathbb{N} \underset{\delta^{*}}{\curvearrowright} \mathbf{C}(\mathscr{X})$, where $\mathbf{C}(\mathscr{X})$ denotes the Banach algebra of all continuous real-valued functions on the underlying state space $\mathscr{X}$ of $\tau \mathbb{N} \underset{\delta}{\curvearrowright} \mathscr{X}$ (interpreted as the target system's algebra of continuous quantities), and the induced linear (dynamical) transition map $\delta^{*}: \tau \mathbb{N} \times \mathbf{C}(\mathscr{X}) \longrightarrow$ $\mathbf{C}(\mathscr{X})$ is defined by $\delta^{*}(\tau k, f)={ }_{d f} g$ with $g(\mathrm{x})=f(\delta(\tau k, \mathrm{x}))$ for all $\mathrm{x}$. The topology in the algebra $\mathbf{C}(\mathscr{X})$ is obtained from the uniform norm $\|f\|={ }_{d f} \sup _{\mathrm{x} \in \mathscr{X}}|f(\mathrm{x})|$.

The same algebraicizing idea works also in the universe dynamical von Neumann algebras. Specifically, to each measurable dynamical model $\tau \mathbb{N} \underset{\delta}{\curvearrowright}(\mathscr{X}, \mathscr{F}, P$ ) (where $P$ is usually the Lebesgue probability measure on $\mathscr{X}$ ) there corresponds a unique dynamical von Neumann algebra model $\tau \mathbb{N} \underset{\delta^{*}}{\curvearrowright}\left(\mathfrak{L}_{\infty}(\mathscr{X}, \mathscr{F}, P), \mathscr{E}_{P}\right)$ such that 
(i) $\mathfrak{L}_{\infty}(\mathscr{X}, \mathscr{F}, P)$ is the von Neumann algebra of essentially bounded real-valued measurable functions on state space $\mathscr{X}$, modulo probability measure $P$ zero, with its induced state $\mathscr{E}_{P}$ in $\mathscr{S}\left(\mathfrak{L}_{\infty}(\mathscr{X}, \mathscr{F}, P)\right)$, and

(ii) the transition map $\delta^{*}: \tau \mathbb{N} \times \mathfrak{L}_{\infty}(\mathscr{X}, \mathscr{F}, P) \longrightarrow$ $\mathfrak{L}_{\infty}(\mathscr{X}, \mathscr{F}, P)$ is defined as before, by $\left[\delta^{*}(\tau k, f)\right](\mathrm{x})=$ $f(\delta(\tau k, \mathrm{x}))$ for all $\mathrm{x}$, where $f \in \mathfrak{L}_{\infty}(\mathscr{X}, \mathscr{F}, P)$. Importantly, $\delta^{*}$ preserves the probabilistic state $\mathscr{E}_{P}$, i.e., we have $\mathscr{E}_{P} \circ \delta^{*}(\tau k, \cdot)=\mathscr{E}_{P}$ for all discrete times $\tau k$ in $\tau \mathbb{N}$.

Thanks to Gelfand and Riesz representation theorems, there is also a reverse construction that transforms algebraic dynamical models back into topological or measurable dynamical models. There are several other important relations between topological (or measurable) dynamical models and algebraic dynamical models, but in this subsection we shall focus only on the problem of algebraic discretization.

In particular, we recall the ever-present pair of projective discretization and injective dediscretization maps between a given continuum parent dynamical model and its descent spatially $\varepsilon$-discretized dynamical model

$$
(\tau \mathbb{N} \underset{\delta}{\curvearrowright} \mathscr{X}) \underset{\mathfrak{I}_{\varepsilon}}{\stackrel{\mathfrak{R}_{\varepsilon}}{\rightleftarrows}}\left(\tau \mathbb{N} \underset{\delta_{\varepsilon}}{\curvearrowright} \mathscr{X}_{\varepsilon}\right),
$$

discussed in the previous subsection. Note that because for compact spaces $\mathscr{X}$ the set $\mathscr{X}_{\varepsilon}$ is a finite discrete (lattice, grid) state space with spacing $\varepsilon>0$, the associated Banach algebra $\mathbf{C}\left(\mathscr{X}_{\varepsilon}\right)$ is finite-dimensional, comprised of vectors or diagonal matrices of dimension $\left|\mathscr{X}_{\varepsilon}\right|$, and likewise for $\mathfrak{L}_{\infty}\left(\mathscr{X}_{\varepsilon}, \mathscr{F}_{\varepsilon}, P_{\varepsilon}\right)$ (where $P_{\varepsilon}$ is specified by the Lebesgue probability of $\varepsilon$-cells). Regarding the empirical interpretation of 'observables' in $\mathbf{C}\left(\mathscr{X}_{\varepsilon}\right)$, we may assume that they are evaluated on $\mathscr{X}_{\varepsilon}$ in terms of dial readings on appropriate measuring instruments, interacting with the target system. The values of discretized quantities in a descent von Neumann algebra model $\mathfrak{L}_{\infty}\left(\mathscr{X}_{\varepsilon}, \mathscr{F}_{\varepsilon}, P_{\varepsilon}\right)$ can given by the averages of corresponding parent dynamical model quantities over $\mathscr{X}_{\varepsilon}$-cells. Having reached this stage, we can now consider ways of lifting the discretization $\mathfrak{R}_{\varepsilon}$ and dediscretization $\mathfrak{I}_{\varepsilon}$ maps from topological models to their Gelfand-given algebraic counterparts $\mathfrak{R}_{\varepsilon}: \mathbf{C}(\mathscr{X}) \longrightarrow \mathbf{C}\left(\mathscr{X}_{\varepsilon}\right)$ and $\mathfrak{I}_{\varepsilon}: \mathbf{C}\left(\mathscr{X}_{\varepsilon}\right) \longrightarrow \mathbf{C}(\mathscr{X})$, respectively. It should be clear that this 'lifting procedure' may be rephrased also in terms of von Neumann algebras.

As we have already remarked in Subsection 3.2, the main obstruction to providing workable algebraic discretization schemes lies in finding convenient discretization and dediscretization maps that support an asymptotic or limit correspondence between the continuum algebraic model $\tau \mathbb{N} \curvearrowright \delta^{*}$ $\mathbf{C}(\mathscr{X})$ and its sequence of descent discretized algebraic models $\tau \mathbb{N} \underset{\delta_{\varepsilon}^{*}}{\curvearrowright} \mathbf{C}\left(\mathscr{X}_{\varepsilon}\right), \tau \mathbb{N} \underset{\delta_{\varepsilon^{\prime}}^{*}}{\curvearrowright} \mathbf{C}\left(\mathscr{X}_{\varepsilon^{\prime}}\right), \ldots$, as their ordered sequence $\varepsilon>\varepsilon^{\prime}>\ldots$ of spacing parameters goes to zero. It turns out that the much-studied pair $\left(\mathfrak{R}_{\varepsilon}, \mathfrak{I}_{\varepsilon}\right)$ in the discretiza- tion/dediscretization relations

$$
\begin{aligned}
& \mathbf{C}(\mathscr{X}) \underset{\mathfrak{I}_{\varepsilon}}{\stackrel{\Re_{\varepsilon}}{\rightleftarrows}} \mathbf{C}\left(\mathscr{X}_{\varepsilon}\right) \\
& \mathfrak{L}_{\infty}(\mathscr{X}, \mathscr{F}, P) \underset{\mathfrak{I}_{\varepsilon}}{\stackrel{\mathfrak{R}_{\varepsilon}}{\rightleftarrows}} \mathfrak{L}_{\infty}\left(\mathscr{X}_{\varepsilon}, \mathscr{F}_{\varepsilon}, P_{\varepsilon}\right)
\end{aligned}
$$

satisfies the definitions of a channel and cochannel, respectively, so that, in particular, the map

$$
\mathscr{S}\left(\mathfrak{L}_{\infty}(\mathscr{X}, \mathscr{F}, P)\right) \stackrel{\mathfrak{I}_{\varepsilon}^{*}}{\longrightarrow} \mathscr{S}\left(\mathfrak{L}_{\infty}\left(\mathscr{X}_{\varepsilon}, \mathscr{F}_{\varepsilon}, P_{\varepsilon}\right)\right)
$$

is a channel. Since the treatment of discretization works best in von Neumann algebra settings, we shall devote the rest of this subsection to the study of discretization of measurable quantities.

In the case of von Neumann dynamical models, the discretization map $\mathfrak{R}_{\varepsilon}: \mathfrak{L}_{\infty}(\mathscr{X}, \mathscr{F}, P) \longrightarrow \mathfrak{L}_{\infty}\left(\mathscr{X}_{\varepsilon}, \mathscr{F}_{\varepsilon}, P_{\varepsilon}\right)$ sends each quantity $f$ to its average on the associated cell of each lattice state in $\mathscr{X}_{\varepsilon}$. By way of illustration, we return to the pendulum example discussed in the previous subsection and for each measurable quantity $f$ we set

$$
\mathfrak{R}_{\varepsilon}(f)={ }_{d f} f_{\varepsilon}: \mathscr{X}_{\varepsilon} \longrightarrow \mathbb{R}
$$

to be a discrete function specified by $f_{\varepsilon}(\varepsilon p, \varepsilon v)={ }_{d f}$ $\int_{\ominus_{\varepsilon,(p, v)}} f(x) P(\mathrm{~d} x)$. As indicated earlier, at each discrete point $(\varepsilon p \varepsilon v)$, the value of function $f_{\varepsilon}$ is obtained by the average of $f$ on the cell determined by the point. It is easy to check that $\mathfrak{R}_{\varepsilon}$ is a linear positive unital map. Now, since the discretized quantity $f_{\varepsilon}$ of $f$ remains real-valued, its measurements discretize its values by a suitable value round-off operation on $\operatorname{Val}(f)$.

The dediscretization map $\mathfrak{I}_{\varepsilon}: \mathfrak{L}_{\infty}\left(\mathscr{X}_{\varepsilon}, \mathscr{F}_{\varepsilon}, P_{\varepsilon}\right) \longrightarrow$ $\mathfrak{L}_{\infty}\left(\mathscr{X}, \mathscr{F}_{\varepsilon}, P\right)$ is defined by a cellwise interpolation of each discrete quantity. That is to say, each measurable quantity $\mathfrak{I}_{\varepsilon}\left(f_{\varepsilon}\right)=f$ is obtained by setting $f(\mathrm{x})={ }_{d f} f_{\varepsilon}\left(\ominus_{\varepsilon},(p, v)\right)$, where $\bullet_{\varepsilon,(p, v)}$ is a cell in $\mathscr{X}_{\varepsilon}$ containing $\mathrm{x}$. Of course, there are several other choices. The goal of algebraic discretization is to find a convenient discretization-dediscretization pair $\left(\mathfrak{R}_{\varepsilon}, \mathfrak{I}_{\varepsilon}\right)$ such that

$$
\lim _{\varepsilon \rightarrow 0}\left\|\mathfrak{I}_{\varepsilon} \circ \mathfrak{R}_{\varepsilon}(u)-u\right\|_{\infty}=0
$$

holds for all quantities $u$ in $\mathfrak{L}_{\infty}(\mathscr{X}, \mathscr{F} \varepsilon, P)$ and the diagram

$$
\begin{array}{cc}
\mathfrak{L}_{\infty}\left(\mathscr{X}, \mathscr{F}_{\varepsilon}, P\right) \stackrel{\mathfrak{d}_{\tau k}}{\longrightarrow} & \mathfrak{L}_{\infty}\left(\mathscr{X}, \mathscr{F}_{\varepsilon}, P\right) \\
\mathfrak{R}_{\varepsilon} \downarrow & \uparrow \mathfrak{I}_{\varepsilon} \\
\mathfrak{L}_{\infty}\left(\mathscr{X}_{\varepsilon}, \mathscr{F}_{\varepsilon}, P_{\varepsilon}\right) \stackrel{\mathfrak{d}_{\tau k}^{\varepsilon}}{\longrightarrow} & \mathfrak{L}_{\infty}\left(\mathscr{X}_{\varepsilon}, \mathscr{F}_{\varepsilon}, P_{\varepsilon}\right)
\end{array}
$$

linking descent discretized dynamics with its parent continuum dynamics commutes in the limit, meaning that the identity

$$
\lim _{\varepsilon \rightarrow 0}\left\|\mathfrak{I}_{\varepsilon} \circ \mathfrak{d}_{\tau k}^{\varepsilon} \circ \Re(f)-\mathfrak{d}_{\tau k}(f)\right\|_{\infty}=0
$$


holds for all quantities $f$ and discrete time instants $\tau k$. Intuitively, the foregoing requirement states that as a descent dis-

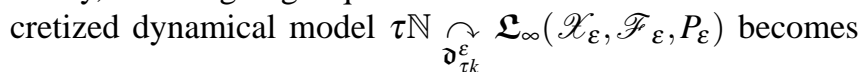
finer and finer under gradually smaller choices of parameter $\varepsilon$, it will approximate the dynamical behavior of its parent continuum dynamical model $\tau \mathbb{N} \underset{\mathcal{f}_{\varepsilon}}{\curvearrowright} \mathfrak{L}_{\infty}(\mathscr{X}, \mathscr{F}, P)$ better and $\mathfrak{d}_{\tau k}^{\varepsilon}$

better, and in the limit, as $\varepsilon$ goes to 0 , the approximation will be perfect, modulo probability measure zero.

In [3], Fabio Benatti and Valerio Cappellini have noted that the foregoing limit of spatial discretization crucially depends on the presence of a logarithmic time scale of temporal discretization. Concretely, in the ambience of a concrete dynamical von Neumann algebra of measurable quantities on a twodimensional continuum torus state space they have shown that the following double-limit theorem

$$
\lim _{\varepsilon \rightarrow 0} \lim _{\substack{\tau k \rightarrow \infty \\ \tau k<\log \frac{1}{\varepsilon}}}\left\|\mathfrak{d}_{\tau k}^{\varepsilon} \circ \mathfrak{R}_{\varepsilon}(u)-\mathfrak{R}_{\varepsilon} \circ \mathfrak{d}_{\tau k}(u)\right\|_{\infty}=0
$$

holds for all quantities $u$ in the dynamical von Neumann algebra model $\mathbb{Z} \underset{\delta^{*}}{\curvearrowright}\left(\mathfrak{L}_{\infty}\left(\mathbb{C}^{2}, \Lambda\right), \mathscr{E}_{\Lambda}\right)$, where $\mathbb{C}^{2}$ is the twodimensional donut-shaped $\mathbb{C} \times \mathbb{C}$ torus space, $\Lambda$ is the Lebesgue probability measure on it and the dynamical transition map $\delta^{*}$ is specified by some simple equations.

Although the significance of the double limit result above is smaller than a discretization specialist might have hoped, it nevertheless indicates an important relationship between spatial and temporal discretizations. Informally, this remarkable result says that if the time evolution of the descent discretized dynamical model is constrained by the exponential inequality of the form $\mathbf{e}^{\tau k}<\frac{1}{\varepsilon}$, stating that as long as the investigator does not go too far into the future in making predictions - so that the necessary spatial discretization refinement by choosing a sufficiently small $\varepsilon$ can be guaranteed - a suitable form of commutativity between discretization and dynamics actually holds. One would hope for similar results to be valid for general dynamical von Neumann algebras. The problem lies, as hinted at earlier, in the correct choice of the pair $\left(\mathfrak{R}_{\varepsilon}, \mathfrak{I}_{\varepsilon}\right)$.

\section{INFORMATION CHANNEL PERSPECTIVE ON MEASUREMENT}

In this section we take up the discussion of the channeltheoretic approach to measurement, begun in Subsection 2.3. As we observed there, measurement can conveniently be characterized in terms of channel-cochannel pairs, relating system and instrument quantity algebras.

Recall again that a classical measurement process involves (i) a measurand of a physical system of interest with a definite but unknown value, and (ii) a correctly calibrated measuring instrument (appropriately chosen for the measurand) - presumed to be dynamically coupled with the physical system for a certain period of time, until an (approximate) equilibrium state is reached. Now, if the coupling and the resulting physical interaction are of the proper kind, then the numerical value read from the measuring instrument's pointer module will be found to be strongly correlated with the measurand's objective value, as it existed immediately prior to the act of measurement. In a successful measurement process, a combined initial 'system + instrument' state (consisting of the system's unknown pre-measurement state and the instrument's known initial 'null position' state) evolves into a unique correlated (entangled) composite post-measurement state, determined by the joint 'system + instrument' physical dynamics. A subsystem restriction of this joint state to the instrument completely determines the pointer quantity's outcome, from which the measurand's real value can then be reconstructed or estimated. Specifically, suppose the physical response of the measuring instrument is given by the direct model equation $\downarrow f=\boldsymbol{E}(f, \ldots)$, where $f$ denotes the measurand and $\downarrow f$ stands for the 'response signal'. ${ }^{36}$ Now, the final outcome of measurement, specified by the values of the instrument's pointer quantity $\oslash f$ - providing support for the measurand's estimation or reconstruction - is obtained from the values of the response quantity $\downarrow f$, using a variant of the calibration equation $\oslash f=\boldsymbol{f}^{\prime}(\not f, \ldots)$. Needless to add, the pointer quantity (D) $f$ serves also as an interface between the instrument and a human observer.

In the spirit of the so-called Heisenberg picture (understood to be a description of natural dynamical systems in terms of Banach or von Neuman algebras of quantities rather than in terms of probabilistic state spaces) suppose the target physical system $\mathrm{S}$ is described by a Banach (von Neumann) algebra

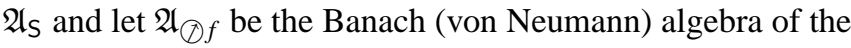
instrument's pointer module (designed to provide measurement outcomes for measurand $f$, instantiated by the system S) with output quantity $\oslash f$.

In modeling the temporal dynamics of the measurement process, the modeler must come up with a dynamical description of not only the (uncoupled) measured $\mathrm{S}$ and measuring $\mathrm{M}_{\oslash f}$ system, but also a complete description of their physical interaction during the measurement process that involves an exchange of energy and directed information flow. Let us assume that the respective dynamical Banach (von Neumann) algebras of the independently prepared target system and pointer module are $\mathbb{T} \underset{\mathfrak{d}}{\curvearrowright} \mathfrak{A}_{\mathrm{S}}$ and $\mathbb{T} \underset{\mathfrak{d}^{\prime}}{\curvearrowright} \mathfrak{A}_{\oslash} \cdot{ }^{37}$ Finally, let the joint dynamics of the 'system + instrument' bipartite system (coupled together at time $t=0$ ) be captured by the family of time-indexed transition maps of the form

$$
\mathfrak{A}_{\mathrm{S}} \otimes \mathfrak{A}_{\oslash f} \stackrel{\mathfrak{m}_{t}}{\longrightarrow} \mathfrak{A}_{\mathrm{S}} \otimes \mathfrak{A}_{\oslash f}
$$

involving the systems' energy quantities and energy flow. Then, as indicated above, the compound system's statistical

\footnotetext{
${ }^{36}$ Note that here we deliberately use the lightning symbol $\& f$ to denote the instrument's internal response quantity as a reminder that in practice mediating quantities tend to be electrical or optical in nature, and the ellipsis in the law serves as a place-holder for unspecified auxiliary quantities. For example, in many instruments the transducer comes with an additional input quantity, capturing environmental interaction, bias, control or modulation.

${ }^{37}$ As before, the transition map's indexing domain $\mathbb{T}$ is a time monoid, usually isomorphic to the additive monoid $\left\langle\mathbb{R}_{+}, 0,+\right\rangle$ of non-negative reals.
} 
state considered at time $t=0$, encoded by $\mathscr{E}_{0}$, evolves in accordance with the earlier defined dual (state) transition map

$$
\mathscr{S}\left(\mathfrak{A}_{\mathrm{S}} \otimes \mathfrak{A}_{\oslash f}\right) \stackrel{\mathfrak{m}_{t}^{*}}{\longrightarrow} \mathscr{S}\left(\mathfrak{A}_{\mathrm{S}} \otimes \mathfrak{A}_{\oslash f}\right),
$$

into a unique state at time $t>0$, denoted by the expectation functional $\mathscr{E}_{t}=\mathfrak{m}_{t}^{*}\left(\mathscr{E}_{0}\right)$.

In more detail, during the interaction process, an unknown pre-measurement system state $\mathscr{E}_{0}$ the experimenter wants to learn about together with the instrument's known 'initial null' state $\mathscr{E}_{f}^{0}$ at time $t=0$, specifying the input product state by the affine lifting (extension) channel $\mathbf{J}^{*}\left(\mathscr{E}_{0}\right)={ }_{d f} \mathscr{E}_{0} \otimes \mathscr{E}_{f} \cdot{ }^{38}$ In accordance with the dynamics of 'system + instrument' composite (determined by the physical laws of interaction), the input product state evolves into a correlated compound state $\mathscr{E}_{t}=\mathfrak{m}_{t}^{*}\left(\mathscr{E}_{0} \otimes \mathscr{E}_{f}^{0}\right)$ considered at time instant $t>0$. The last step - as it should be apparent by now - is provided by the so-called restriction channel $\mathbf{R}^{*}$, defined by the state restriction $\left[\mathbf{R}\left(\mathscr{E}_{t}\right](v)=\mathscr{E}_{t}(1 \otimes v)\right.$ for any instrument quantity $v$ in $\mathfrak{A}_{\oslash f}(\oslash f)$.

At the root of interactive (instrument-based) measurement processes is the following fundamental commutative square (and its obvious dual):

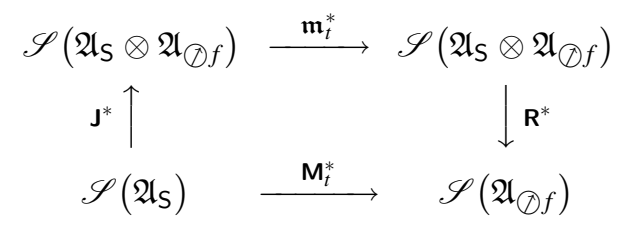

Upon examining the diagram, we see that the system-toinstrument measurement channel $\mathbf{M}_{t}^{*}$ is specified by the composite $\mathbf{M}_{t}^{*}={ }_{d f} \mathbf{R}^{*} \circ \mathfrak{m}_{t}^{*} \circ \mathbf{J}^{*}$ for all moments of time $t$.

One reason why instrument-based measurement theory appears to be so complex is because it deals with structures at two distinct levels, one of which complements or supervenes on the other. Structures at the most fundamental level - enjoying a great deal of attention by physicists in view of being essential to the physical understanding all classical measurement processes - are given by the underlying physical interactions between the measured and measuring systems. In the pursuit of this ground level structure, measurement theorists regularly take concepts from physics and systems theory, and apply them to various measuring instruments and measurement processes. For us, this level requires least comment, since it has been covered already, based on [5].

The second level of structure is best seen in the formal embodiment of a functionalist view of measurement. As is by

\footnotetext{
${ }^{38}$ A lifting channel of the form $\mathbf{J}^{*}: \mathfrak{A}_{\mathrm{S}} \longrightarrow \mathfrak{A}_{\mathrm{S}} \otimes \mathfrak{A}_{\oslash f}$ is said to be nondemolitionist for the system state $\mathscr{E}$ (or $\mathscr{E}$ is invariant with respect to $\mathbf{J}^{*}$ ) provided that $\left[\mathbf{J}^{*}(\mathscr{E})\right](u \otimes I)=\mathscr{E}(u)$ holds for all system quantities $u$. Nondemolitionist channels characterize measurement interactions that do not alter the system's initial state. We know that various measurement processes tend to change the measured system. For example, active sensors (e.g, radars and sonars) add energy to the 'system + instrument' environment as part of the measurement process, and electrical instruments usually draw energy from the measured system, causing a loading error. Most chemical measurements damage or destroy at least part of the analyzed substance, and so forth.
}

now well known, in practice the measurand (e.g., mass, temperature, energy, and so forth) of a target system can be measured in many distinct and competing ways, and with varying degrees of accuracy, employing different physical methods and principles, and yet, by and large, the resulting quantity of information obtained about the target system in the corresponding measurement outcomes is, modulo some errors, essentially the same. Thus, if what matters is only the transfer of information about the measurand from its instantiating target system to the instrument's pointer module, where it is received in the form of the instrument's pointer quantity value to be read off later, and not the specific physical mechanism underlying the measurement process, then it is more effective to work with a considerably simpler channel-theoretic model of measurement, based on quantity and state channels. In information-theoretic frameworks it is completely irrelevant which type of measuring instrument is used to perform a measurement and which physical laws are involved. This higherlevel (supervening) approach to measurement processes is extremely convenient, because it is relatively easy to transform information between different types of physical carriers (such as electric currents in wires, light pulses in optical fibers, and so on). Furthermore, errors, perturbations and possible losses of information occurring during transmission are also easily tractable. The viewpoint we adopt next is to regard measurement as a transfer of information from the target system to the measurand's pointer module. ${ }^{39}$

As we have already seen, from the standpoint of algebraicanalytic measurement theory, the basic strategy is to represent each step in a measurement process by a suitable channelcochannel pair, in which the cochannel maps all quantities of an input (source) system M (usually a measurement apparatus), described by (say) a Banach algebra $\mathfrak{A}_{M}$, to suitable estimator quantities of an output (receiver) system $\mathrm{S}$, in general represented by a different Banach algebra $\mathfrak{A}_{\mathrm{S}}$.

Our aim here is to model the measurement process channeltheoretically (without invoking any details regarding the physics of measurement coupling), usually by a cochannel $M$ which maps the pointer module's measurement quantities in $\mathfrak{A}_{\mathrm{M}}$ to those quantities in the system's algebra $\mathfrak{A}_{\mathrm{S}}$ which serve as optimal estimators for the system's quantities, given the instrument's information about $S$ embodied in the pointer module of $\mathfrak{A}_{\mathrm{M}}$. Each cochannel $M$ captures a particular way in which a measuring system encodes information about the measured system. Conditionally upon the results of continuous measurement, a cochannel minimally revises and updates a prior quantitative information about the system's quantities.

\footnotetext{
${ }^{39}$ Here we assume that information is not a concrete thing or substance that is transported like furniture from one place to another; it is not part of the material contents of the world, located somewhere in space. Rather, information is a fundamental theoretical entity (in the sense of Hans Reichenbach's $a b$ stracta), representing that which is brought into existence by an information source and required to be reproducible at the receiver end of a communication channel, if the transmission is to be regarded as a success. Its roundabout description 'that which is brought into existence' is formally analyzed in terms of (continuous, measurable, etc.) sequences of probabilistic states.
} 


\subsection{Discrete measurement of angular positions of a physi- cal pendulum}

There are at least three major categories of classical measurement. Historically, the earliest types of measurement were those based on the principle of direct comparison. We have already met some of these in Subsection 2.3 and called them static measurements. Indirect or interactive instrument-based measurements dominate modern measurement practices. As well known, these measurements come in two major flavors: discrete-time and continuous-time dynamical measurements. We shall have space to consider only one example of a discrete-time dynamical measurement.

Suppose we wish to measure the angular positions of a simple pendulum's bob at various instants of time. We have already seen in Subsection 2.1.1 that in the case of instrumentbased measurement of pendulum positions the starting point is the Banach algebra $\mathfrak{A}(p, v)$, generated by position $p$ and velocity $\vee$ quantities, and Gelfand-isomophic to the concrete Banach algebra of observables $\mathbf{C}(\operatorname{Val}(p) \times \operatorname{Val}(v)) \cong$ $\mathbf{C}(\operatorname{Val}(\mathrm{p})) \otimes \mathbf{C}(\operatorname{Val}(\mathrm{v}))$. Since the value space $\operatorname{Val}(\mathrm{p})$ is equal to the circle group $\mathbb{C}=\mathbb{R}_{/ 2 \pi \mathbb{Z}}$ of reals and $\operatorname{Val}(\mathrm{v})=[0, V]$ for some upper limit $V$ of angular velocity that does not exceed the speed of light, we quickly obtain our start-up dynamical Banach algebra $\mathbb{R}_{+} \curvearrowright \mathbf{d}(\mathbb{C}) \otimes \mathbf{C}([0, V])$ (together with the algebra isomorphism $\mathfrak{A}(\mathrm{p}, \mathrm{v}) \cong \mathbf{C}(\mathbb{C}) \otimes \mathbf{C}([0, V]))$, intended for modeling the pendulum's dynamical behavior. As shown in [5] and reviewed in Subsection 2.5, the transition map of this dynamical Banach algebra is derived from the solution space of classical pendulum equations. Here, as before, the dynamical Banach algebra represents the temporal evolution of all quantities that have been found to be decisive in a complete description of the pendulum's motion under consideration, without any regard for measurement interaction.

In order to meet the requirements of discrete measurement, we need a discrete variant of the above-discussed parent dynamical Banach algebra. To obtain the descent discrete-time dynamical Banach algebra $\tau \mathbb{N} \overbrace{\mathfrak{d}_{\tau}} \mathbf{C}(\mathbb{C}) \otimes \mathbf{C}([0, V])$, we use the temporal discretization method of Subsection 3.1. By construction, in this discrete algebraic model the pendulum's angular position and velocity values are available only at discrete moments of time, referred to by $\tau k$ (integer $k$ time units of a chosen time-step $\tau$ ).

We now turn our attention to position measurement. Since pendulums are among the most studied physical systems, there are several known ways of measuring their positions and velocities. In discrete position measurements the most common methods are the so-called stroboscopic approaches. In a typical experimental set-up, position measurement is based on the variation of a suitably positioned light-dependent resistor, located proximally to the pendulum's plane of motion, whose electrical resistance abruptly changes when the pendulum's moving bob cuts the path of the light from a laser. In one implementation, equally spaced laser beams are directed perpendicularly to the pendulum's plane of motion, with electric sensors on its opposite side. Design specifics of this and related position measurement methods may be found in [9] and the references therein. Since we may know the response of this stroboscopic measuring system without knowing the technical details of its optical and electrical dynamics, we shall assume that the value space $\operatorname{Val}(\oslash p)$ of pointer quantity $\oslash p$ (calibrated in angular or length units) of $p$ is a subset of discrete points in $\mathbb{C}$. This means that since the pendulum's positions are measured at discrete time instants stroboscopically with limited accuracy, we need a spatial discretization of the circle group $\mathbb{C}$ of positions, specified by the discrete set $\mathbb{C}_{\varepsilon}={ }_{d f}$ $\varepsilon \mathbb{N} \cap \mathbb{C}=\{0, \varepsilon, 2 \varepsilon, \cdots\}$ of possible angular pendulum positions on a circle, where $\varepsilon>0$ is a fixed discretization parameter, chosen in arc or length units, e.g., one arc second or one millimeter. We can now define the space-time parametrized discretization map $\Re_{\tau, \varepsilon}: \mathbb{R}_{+} \times \mathbb{C} \longrightarrow \tau \mathbb{N} \times \mathbb{C}_{\varepsilon}$ as in Subsection 2.3, by setting $\Re_{\tau, \varepsilon}(t, \theta)=_{d f}\left(\left\lceil\frac{t}{\tau}+\frac{1}{2}\right\rceil \cdot \tau,\left\lceil\frac{\lambda}{\varepsilon}+\frac{1}{2}\right\rceil \cdot \varepsilon\right)$. Here the basic idea is to assign to each ideal angular position value $\theta$ at a given ideal time point $t$ the unique discrete position that is closest to $\theta$ - measured stroboscopically - at the discrete time nearest to $t$.

Moving on to the 'system + instrument' state dynamics

$$
\left.\left.\mathscr{S}\left(\mathbf{C}(\mathbb{C} \times[0, V]) \times \mathbb{C}_{\varepsilon}\right)\right) \stackrel{\mathfrak{m}_{t}^{*}}{\longrightarrow} \mathscr{S}\left(\mathbf{C}(\mathbb{C} \times[0, V]) \times \mathbb{C}_{\varepsilon}\right)\right),
$$

note that because the pendulum's state of motion is not affected by measurement, we may set for deterministic states $\mathfrak{m}_{t}\left(\theta_{0} \otimes v_{0} \otimes \theta_{\tau 0}^{\prime}\right)=\theta_{t} \otimes v_{t} \otimes \Re_{\tau, \varepsilon}\left(t, \theta_{t}\right)$, where the tensor component for position measurement changes from its initial value to the next discrete value, established by stroboscopic measurement.

By exactly the same reasoning used in the previous subsection, we use the fundamental contravariant commutative square

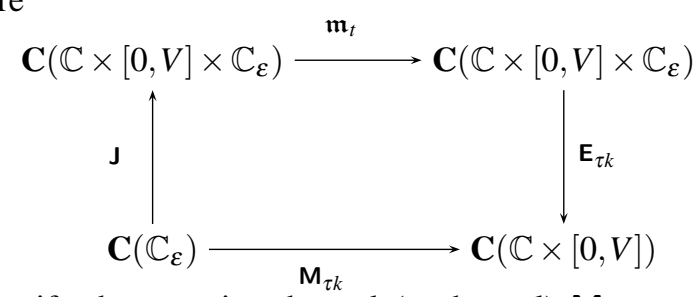

to specify the quantity channel (cochannel) $\mathbf{M}_{\tau k}={ }_{d f} \mathbf{E}_{\tau k} \circ$ $\mathfrak{m}_{t} \circ \mathbf{J}$, intended for modeling a discrete measurement of the target pendulum's positions. As before, we set $\mathbf{J}(v)=1 \otimes 1 \otimes$ $u$ for each pendulum quantity $u$. Since the dynamics of the 'system + instrument' does not alter the pendulum's states, we define the right most cochannel $\mathbf{E}_{\tau k}$ in the square for all observables $\xi$ in $\mathbf{C}\left(\mathbb{C} \times[0, V] \times \mathbb{C}_{\varepsilon}\right)$ by $\left[\mathbf{E}_{\tau k}(\xi)\right]\left(\theta_{t}, v_{t}, \theta_{\tau k}^{\prime}\right)=$ $\theta_{\tau k}^{\prime}$

The channel $\mathbf{M}_{\tau k}^{*}$ maps the pendulum's position-velocity deterministic $\operatorname{state}\left(\theta_{t}, v_{t}\right)$ at time $t$ to the measured position value $\theta_{\tau k}^{\prime}$ at discrete time $\tau k$, nearest to $t$. This discrete value, in turn, can be used to specify the value of the position estimator $\widehat{p}$. We do not have space to treat discrete position measurement with random error. More general forms of position measurement require the cochannel $\mathbf{E}_{\tau k}$ to be a conditional expectation. (Full details on conditional expectations in this context are given in [10] and references therein.) 


\subsection{Information channels from measured systems to mea- suring instruments}

Before turning to the explicit details of application of state channels and their cochannels to measurement problems, it is important to point out that in the case of quantity channels of form $\mathbf{M}_{f}: \mathfrak{A}_{\oslash f} \longrightarrow \mathfrak{A}_{\mathrm{S}}$ for measuring quantity $f$, no account is taken of the measurement interaction between the target system $\mathrm{S}$ and the selected measuring instrument for $f$. The formal framework in which the instrument-based measurement interaction problem (and many problems like it) can be framed is given by the so-called conditional expectation cochannel, having the form

$$
\mathfrak{A}_{\mathrm{S}} \otimes \mathfrak{A}_{\oslash f} \stackrel{\mathbf{E}}{\longrightarrow} \mathfrak{A}_{\mathrm{S}}
$$

whose dual state channel $\mathbf{E}^{*}$ transfers the system's extant (statistical) state to a correlated state of the composite 'system + instrument' bipartite system, from which the instrument's final state is determined by a subsystem reduction. More particularly, as we have seen, the state channel of a measurement process is modeled by the following cascade of information channels:

$$
\mathscr{S}\left(\mathfrak{A}_{\mathrm{S}}\right) \stackrel{\mathbf{E}^{*}}{\longrightarrow} \mathscr{S}\left(\mathfrak{A}_{\oslash f} \otimes \mathfrak{A}_{\mathrm{S}}\right) \stackrel{\mathfrak{m}_{t}}{\longrightarrow} \mathscr{S}\left(\mathfrak{A}_{\oslash f} \otimes \mathfrak{A}_{\mathrm{S}}\right) \stackrel{\mathbf{R}}{\rightarrow} \mathscr{S}\left(\mathfrak{A}_{\oslash f}\right)
$$

The channel diagram above shows how to obtain knowledge about the unknown pre-measurement state of the system from a known correlated post-measurement state of the instrument, after the measurement interaction has reached its equilibrium.

The quantity channel $\mathbf{E}: \mathfrak{A}_{\mathrm{S}} \otimes \mathfrak{A}_{\oslash f} \longrightarrow \mathfrak{A}_{\mathrm{S}}$ turns out to be extremely important in handling the information-theoretic aspects of measurement processes. It is easy to verify that its Gelfand representation is given an averaging operator of the form

$$
\mathbf{C}\left(\mathscr{X}_{\mathrm{S}} \times \mathscr{X}_{\oslash f}\right) \cong \mathbf{C}\left(\mathscr{X}_{\mathrm{S}}\right) \times \mathbf{C}\left(\mathscr{X}_{\oslash f}\right) \stackrel{\widehat{\mathbf{E}}}{\longrightarrow} \mathbf{C}\left(\mathscr{X}_{\mathrm{S}}\right),
$$

defined by $[\widehat{\mathbf{E}}(h)]\left(\mathrm{x}=\int_{\mathscr{X}_{\bigoplus f}} h\left(\mathrm{x}, \mathrm{x}^{\prime}\right) T\left(\mathrm{x}, \mathrm{dx} \mathrm{x}^{\prime}\right)\right.$, where $T$ : $\mathscr{X}_{\mathrm{S}} \longrightarrow \mathbf{P}\left(\mathscr{X}_{\bigoplus f}\right)$ is a given transition probability. Thanks to its relationship to averaging, the cochannel $\mathbf{E}$ satisfies the following properties for all quantities $u$ and $v$ on the product state space $\mathscr{X}_{\mathrm{S}} \times \mathrm{X}_{\oslash f}$ :

(i) $\mathbf{E}(1 \otimes 1)=1$;

(ii) $u \geq O \Longrightarrow \mathbf{E}(u) \geq O$;

(iii) $\mathbf{E}(u+v)=\mathbf{E}(u)+\mathbf{E}(v)$;

(iv) $\mathbf{E}(u \cdot \mathbf{E}(v))=\mathbf{E}(u) \bullet \mathbf{E}(v)$.

What is perhaps even more startling than the properties of $\mathbf{E}$ listed above is the fact that cochannels are Banach (von Neumann) algebra analogs of existential quantifiers in distributive lattices. Note that the quantity channel $\mathbf{M}_{f}$ introduced at the beginning of this subsection earlier is obtained from $\mathbf{E}$ by restriction: $\mathbf{M}_{f}(h)=\mathbf{E}(1 \otimes h)$ for all $h$ in $\mathfrak{A}_{\oslash f}$.
We turn now to describing some important measurement processes using the cochannel-channel framework. In Section 2.1 we have already noted the fundamental importance of the cochannel-channel pair

$$
\mathfrak{A}^{\prime} \times \mathscr{S}\left(\mathfrak{A}^{\prime}\right) \stackrel{\left(\mathbf{M}, \mathbf{M}^{*}\right)}{\longrightarrow} \mathfrak{A} \times \mathscr{S}(\mathfrak{A})
$$

between Banach (von Neumann) algebras and their respective state spaces. Let us point out at once that a cascade sequence (important in representing a sequence of measurement sensors, processors, filters, amplifiers, and convertors)

$$
\mathfrak{A}^{\prime \prime} \times \mathscr{S}\left(\mathfrak{A}^{\prime \prime}\right) \stackrel{\left(\mathbf{N}, \mathbf{N}^{*}\right)}{\longrightarrow} \mathfrak{A}^{\prime} \times \mathscr{S}\left(\mathfrak{A}^{\prime}\right) \stackrel{\left(\mathbf{M}, \mathbf{M}^{*}\right)}{\longrightarrow} \mathfrak{A} \times \mathscr{S}(\mathfrak{A})
$$

composes in the usual way into the cochannel-channel pair

$$
\mathfrak{A}^{\prime \prime} \times \mathscr{S}\left(\mathfrak{A}^{\prime \prime}\right) \stackrel{\left(\mathbf{M} \circ \mathbf{N},(\mathbf{M} \circ \mathbf{N})^{*}\right)}{\longrightarrow} \mathfrak{A} \times \mathscr{S}(\mathfrak{A})
$$

The verification is easy, based on the definition of ther cochannel-channel pair. From the foregoing composition operation we obtain a category of cochannel-channel pairs, that can be used as a formal framework for the study of measurement processes. Along similar lines, it is easy to check that two cochannel-channel pairs

$$
\begin{gathered}
\mathfrak{A}^{\prime} \times \mathscr{S}\left(\mathfrak{A}^{\prime}\right) \stackrel{\left(\mathbf{M}, \mathbf{M}^{*}\right)}{\longrightarrow} \underset{A}{\text { and }} \times \mathscr{S}(\mathfrak{A}) \\
\mathfrak{B}^{\prime} \times \mathscr{S}\left(\mathfrak{B}^{\prime}\right) \stackrel{\left(\mathbf{N}, \mathbf{N}^{*}\right)}{\longrightarrow} \mathfrak{B} \times \mathscr{S}(\mathfrak{B})
\end{gathered}
$$

determine their tensor product pair (important in applications to repeated measurement of a single measurand or to a joint measurement of several measurands)

$$
\left(\mathfrak{A}^{\prime} \otimes \mathfrak{B}^{\prime}\right) \times \mathscr{S}\left(\mathfrak{A}^{\prime} \otimes \mathfrak{B}^{\prime}\right) \stackrel{\left(\mathbf{M} \otimes N,(\mathbf{M} \otimes \mathbf{N})^{*}\right)}{\longrightarrow}(\mathfrak{A} \otimes \mathfrak{B}) \times \mathscr{S}(\mathfrak{A} \otimes \mathfrak{B})
$$

There are many other important constructions on channels and cochannels, needed for the description of special measurement processes, including measurement fusion, measurements involving demolition, and continuous measurement that, due to limitations on space and time, must be omitted.

\section{CONCLUSIONS}

In this paper we have presented and developed the framework of Banach and von Neumann algebras for quantities, together with their associated (convex) spaces of expectation functionals, as a comprehensive and unified algebraic-analytic framework for measurement theory. It is our hope that the formal adequacy of this paradigm was made apparent by the remarkable flexibility with which it accommodated mathematically natural constructions, representing various aspects of measurement - such as tensor products of algebras (modeling various couplings of target systems and measuring instruments), 
dynamical algebras (capturing temporal evolutions of quantities instantiated by natural systems), and spaces of expectation functionals defined over algebras (encoding evaluations of quantities through measurement). A striking element of this framework is a large variety of information channels between quantity algebras that model functionally conceived transfers of information from target systems to measuring instruments, and also - in the converse direction from instruments to systems - various estimations and reconstructions of the measurands' values from the outputs of measuring instruments. The framework's mutability also allowed us to model the relations between continuum-based theoretical models and finite/discrete measurement data in terms of increasingly refined discretizations of continuum theoretical models. Finally, we believe that the conceptual adequacy of the algebraicanalytic framework was made apparent by various representation theorems, which connect quantities to the states of modeled natural systems by providing us with deterministic as well as probabilistic interpretations of autonomously specified quantity algebras and information channels between them, in terms of uniquely determined state spaces and observables thereon.

\section{REFERENCES}

[1] Aerts, D., Daubechies, I. (1978). Physical justification for using the tensor product to describe two quantum systems as one joint system. Helvetica Physica Acta, 51, 661-675.

[2] Batitsky, V., Domotor, Z. (2007). When good theories make bad predictions. Synthese, 157, 79-103.

[3] Benatti, F., Cappellini, V. (2005). Continuous limit of discrete sawtooth maps and its algebraic framework. Journal of Mathematical Physics, 46, 1-25.
[4] Domotor, Z., Batitsky, V. (2008). The analytic versus representational theory of measurement: A philosophy of science perspective. Measurement Science Review, 8 (6), 129-146.

[5] Domotor, Z., Batitsky, V. (2009). An algebraic-analytic framework for measurement theory. Under review for publication in Measurement.

[6] Gelfand, I. M. (1939). On normed rings. Doklady Akad. Nauk U.S.S.R. 23, 430-432.

[7] Kadison, R. V., Ringrose, J. R. (1983). Fundamentals of the Theory of Operator Algebras, Vol. II. Academic Press, San Diego.

[8] Lasota, A., Mackey, M. C. (1994). Chaos, Fractals, and Noise. Stochastic Aspects of Dynamics, 2nd edition. Springer-Verlag, New York.

[9] Lima, F. M. S., Arun, P. (2006). An accurate formula for the period of a simple pendulum oscillating beyond the small angle regime. American Journal of Physics, 74, 892-895.

[10] Lindblad, G. (1996). On the existence of quantum subdynamics. Journal of Physics A: Math. Gen., 29, 41974207.

[11] Nassopoulos, G. F. (1999). On a comparison of real with complex involutive complete algebras. Journal of Mathematical Sciences 36, 3755-3765.

[12] Sakai, S. (1998). $\quad C^{*}$-Algebras and $W^{*}$-Algebras. Springer, New York.

[13] Umegaki, H. (1969). Representations and extremal properties of averaging operators, and their applications to information channels. Journal of Mathematical Analysis and Applications, 25, 41-73.

[14] Walters, P. (1981). Introduction to Ergodic Theory. Springer, Tokyo.

[15] Yosida, Y. (1980). Functional Analysis, 6th edition. Springer-Verlag, New York. 